\title{
Importance of lifetime effects in breakup and suppression of complete fusion in reactions of weakly bound nuclei
}

\author{
K. J. Cook, ${ }^{*}$ E. C. Simpson, D. H. Luong, Sunil Kalkal, M. Dasgupta, and D. J. Hinde \\ Department of Nuclear Physics, Research School of Physics and Engineering, The Australian National University, Canberra, \\ Australian Capital Territory 2601, Australia
}

(Received 4 March 2016; revised manuscript received 9 May 2016; published 8 June 2016)

\begin{abstract}
Background: Complete fusion cross sections in collisions of light weakly bound nuclei and high- $Z$ targets show suppression of complete fusion at above-barrier energies. This has been interpreted as resulting from the breakup of the weakly bound nucleus prior to reaching the fusion barrier, reducing the probability of complete charge capture. Below-barrier studies of reactions of ${ }^{9} \mathrm{Be}$ have found that the breakup of ${ }^{8} \mathrm{Be}$ formed by neutron stripping dominates over direct breakup and that transfer-triggered breakup may account for the observed suppression of complete fusion.

Purpose: This paper investigates how the above conclusions are affected by lifetimes of the resonant states that are populated prior to breakup. If the mean life of a populated resonance (above the breakup threshold) is much longer than the fusion time scale, then its breakup (decay) cannot suppress complete fusion. For short-lived resonances, the situation is more complex. This work explicitly includes the mean life of the short-lived $2^{+}$resonance in ${ }^{8} \mathrm{Be}$ in classical dynamical model calculations to determine its effect on energy and angular correlations of the breakup fragments and on model predictions of suppression of cross sections for complete fusion at above-barrier energies.

Method: Previously performed coincidence measurements of breakup fragments produced in reactions of ${ }^{9} \mathrm{Be}$ with ${ }^{144} \mathrm{Sm},{ }^{168} \mathrm{Er},{ }^{186} \mathrm{~W},{ }^{196} \mathrm{Pt},{ }^{208} \mathrm{~Pb}$, and ${ }^{209} \mathrm{Bi}$ at energies below the barrier have been reanalyzed using an improved efficiency determination of the BALiN detector array. Predictions of breakup observables and of complete and incomplete fusion at energies above the fusion barrier are then made using the classical dynamical simulation code PLATYPUS, modified to include the effect of lifetimes of resonant states.

Results: The agreement of the breakup observables is much improved when lifetime effects are included explicitly. Sensitivity to subzeptosecond lifetime is observed. The predicted suppression of complete fusion owing to breakup is nearly independent of $Z$ and has an average value of $\sim 9 \%$. This is below the experimentally determined fusion suppression, which is typically $\sim 30 \%$ in these systems.

Conclusions: Inclusion of resonance lifetimes is essential to correctly reproduce breakup observables. This results in a larger fraction of nuclei remaining intact at the fusion-barrier radius compared with calculations that do not explicitly include lifetime effects. The more realistic treatment of breakup followed in this work leads to the conclusion that the suppression of complete fusion cannot be fully explained by breakup prior to reaching the fusion barrier. Only one-third of the observed fusion suppression can be attributed to the competing process of breakup. Other mechanisms that can suppress complete fusion must therefore be investigated. One of the possible candidates is cluster transfer that produces the same heavy targetlike nuclei as those formed by incomplete fusion.
\end{abstract}

DOI: 10.1103/PhysRevC.93.064604

\section{INTRODUCTION}

The causes of complete fusion suppression in above-barrier reactions with light, weakly bound nuclei is a key question in fusion dynamics. Fusion measurements of ${ }^{9} \mathrm{Be}+{ }^{208} \mathrm{~Pb},{ }^{209} \mathrm{Bi}$ [1-4] and ${ }^{6,7} \mathrm{Li}+{ }^{209} \mathrm{Bi}[2,5]$ show that above-barrier complete fusion cross sections (experimentally defined as capture of the full charge of the projectile) are reduced by $\sim 30 \%$, both in comparison with those predicted by complete fusion models and with measurements for well-bound nuclei forming the same compound nucleus [2,6]. Complete fusion suppression in reactions with ${ }^{9} \mathrm{Be}$ has been observed for a variety of targets in the range $39 \leqslant Z \leqslant 83$ [4,7-10]. This suppression was initially suggested to result from direct breakup of ${ }^{9} \mathrm{Be}(\rightarrow \alpha+\alpha+n)$ prior to reaching the fusion barrier [1].

*kaitlin.cook@anu.edu.au
It was conjectured that breakup reduces the probability of the full charge of the projectilelike nucleus being captured, thus suppressing complete fusion $(\mathrm{CF})$ and increasing the incomplete fusion (ICF) cross sections.

Experiments were undertaken to probe the extent of the role of breakup in complete fusion suppression. These experiments were performed at below-barrier energies to allow clearer investigation of breakup because there is essentially no absorption of the charged fragments [11]. These investigations found that transfer followed by breakup contributes much more than direct breakup to the total breakup probability $[12,13]$. In the case of ${ }^{9} \mathrm{Be}$, breakup in interactions with ${ }^{144} \mathrm{Sm},{ }^{168} \mathrm{Er}$, ${ }^{186} \mathrm{~W},{ }^{196} \mathrm{Pt},{ }^{208} \mathrm{~Pb}$, and ${ }^{209} \mathrm{Bi}$ is dominated by neutron stripping forming ${ }^{8} \mathrm{Be}$, which subsequently breaks up into $\alpha+\alpha$, rather than ${ }^{9} \mathrm{Be}$ undergoing direct breakup into $\alpha+\alpha+n$ or ${ }^{8} \mathrm{Be}+n$.

It was recognized early on [11] that very long-lived states, such as the $0^{+}$ground state of ${ }^{8} \mathrm{Be}$, which has a mean life of $\sim 10^{-16} \mathrm{~s}$ [14], results in breakup far from the targetlike 
nucleus. It therefore cannot contribute to complete fusion suppression. At above-barrier energies, the ${ }^{8} \mathrm{Be}$ nucleus in its ground state will pass inside the fusion barrier and be absorbed before decay can occur. However, the population of broad resonances with much shorter mean lives will result in breakup close to the targetlike nucleus.

The question then is: What is the quantitative contribution of near-target transfer-triggered breakup to the suppression of complete fusion? This was previously addressed by first obtaining breakup probabilities as a function of distance of closest approach ("breakup functions") [12] at below-barrier energies. These breakup functions were then used as input to the classical dynamical model code PLATYPUS $[15,16]$ to predict complete and incomplete fusion cross sections at above-barrier energies [12,15] that agreed satisfactorily with experimental results $[2,4,7,10]$.

In PLATYPUS, the lifetimes of the states populated were not explicitly taken into account. However, locations of breakup and the lifetimes of states are intimately related: Finite but small mean lives will change the positions at which breakup occurs along the trajectory of the nuclei. Indeed, recent work [17] has highlighted that the precise location of breakup relative to the targetlike nucleus is critical to reaction outcomes and, further, that there exist experimental observables that can probe these effects.

In this work, we investigate quantitatively the effect of the lifetime of short-lived resonant states on breakup processes and the resultant incomplete fusion. Measurements of transfer reactions populating ${ }^{8} \mathrm{Be}$ can be completely explained by the population of ${ }^{8} \mathrm{Be}$ in its $0^{+}, 2^{+}$, and, at higher excitations, $4^{+}$ states $[18,19]$. In breakup following ${ }^{7} \mathrm{Li}$ collisions with ${ }^{58} \mathrm{Ni}$, it has been shown that transfer populates the $0^{+}$and $2^{+}$states in ${ }^{8} \mathrm{Be}$ [17]. The 3.03-MeV $2^{+}$state of ${ }^{8} \mathrm{Be}$ has an on-resonance width of $\Gamma\left(E_{R}\right)=1513 \pm 15 \mathrm{keV}$, and thus a mean life of $\tau=\hbar / \Gamma\left(E_{R}\right)=0.44 \times 10^{-21} \mathrm{~s}$ [14]. As such, breakup from this state will occur very close to the targetlike nucleus. To determine the effect on complete fusion, it is then necessary to quantitatively understand whether such short mean lives carry a significant fraction of excited projectilelike nuclei inside the fusion barrier before breakup occurs, thus reducing the suppression of complete fusion owing to breakup.

To address this question, this work presents a reanalysis of the extensive sub-barrier breakup measurements of Rafiei et al. [12], using a modified version of PLATYPUS which incorporates resonance lifetimes. The reanalysis of these experimental data is presented in Sec. II. An improved method has been used to better determine the coincidence detection efficiency of the detector array, discussed in Sec. III. As a result of these changes, a different efficiency correction for the detector geometry has resulted, which feeds back into the determination of the breakup function - the probability of breakup along a trajectory with distance of closest approach $R_{\text {min }}$ - given as input into PLATYPUS. Model sensitivities to breakup observables and the resultant modifications to PLATYPUS are discussed in Sec. IV. New below-barrier breakup functions are derived in Sec. V. The calculations of above-barrier fractions of incomplete fusion are presented in Sec. VI, and the consequences of these calculations for the role of breakup in the suppression of complete fusion is discussed in Sec. VII.

\section{DATA ANALYSIS}

Full experimental details for the data analyzed here can be found in Ref. [12], and a brief summary is given here for completeness. Beams of ${ }^{9} \mathrm{Be}$ at below-barrier energies were delivered by the 14UD electrostatic accelerator at the Australian National University Heavy Ion Accelerator Facility onto isotopically enriched targets of ${ }^{144} \mathrm{Sm},{ }^{168} \mathrm{Er},{ }^{186} \mathrm{~W},{ }^{196} \mathrm{Pt}$, ${ }^{208} \mathrm{PbS}$, and ${ }^{209} \mathrm{Bi}$. The breakup of ${ }^{9} \mathrm{Be}$, whether direct or triggered by neutron stripping, results in two coincident $\alpha$ particles. The Breakup Array for Light Nuclei (BALiN) was used to detect these coincident fragments. The array is composed of four double-sided silicon strip detectors (DSSDs), each with 16 arcs and 8 sectors, resulting in 512 effective pixels over the array. Below-barrier $\left(E / V_{B} \sim 0.65-0.9\right)$ measurements of coincident $\alpha-\alpha$ pairs were made, as reported in Ref. [12], with the goal of extracting breakup probabilities as a function of the distance of closest approach. In analyses such as these, the challenge is in separating coincident breakup events from all other reaction outcomes that result in coincident signals in a detector array. Genuine coincident breakup events were distinguished from spurious coincidence events (mainly resulting from random coincidences between scattered projectiles and electronic noise) by selecting the characteristic diagonal bands that appear when plotting the energy of one coincident particle $\left(E_{1}\right)$ against the energy of the other $\left(E_{2}\right)$ (see, for example, Fig. 3 of Ref. [12]). For completeness, in Appendix A we describe an improved method for removal of spurious coincidence events resulting from cross talk or particles crossing an interstrip partition, which are not removed by $E_{1}-E_{2}$ gating.

\section{A. Distinguishing near-target and asymptotic breakup}

After the removal of spurious events, the reconstructed spectra of reaction $Q$ value against relative energy of the two coincident breakup fragments, $E_{\text {rel }}$, for ${ }^{9} \mathrm{Be}+{ }^{144} \mathrm{Sm},{ }^{168} \mathrm{Er}$, ${ }^{186} \mathrm{~W},{ }^{196} \mathrm{Pt},{ }^{208} \mathrm{~Pb}$, and ${ }^{209} \mathrm{Bi}$ at center-of-mass energy $E_{\mathrm{c} . \mathrm{m}}$. such that $\frac{E_{\text {c.m. }}}{V_{\mathrm{B}}} \sim 0.9$ are shown in Fig. 1. Compared to previous results, at this stage in the reanalysis, the data differ mainly in the larger number of events from ${ }^{8} \mathrm{Be}$ ground-state decay, as noted in Appendix A. The $Q$ values are determined by

$$
Q=\left(E_{1}+E_{2}+E_{\text {recoil }}\right)-E_{\mathrm{lab}},
$$

where $E_{i}$ are the energies of each fragment, corrected for energy loss through the target, mylar foil, aluminum layer, and silicon dead layer; $E_{\text {lab }}$ is the beam energy after traversing half the target thickness; and $E_{\text {recoil }}$ is the energy of the recoiling targetlike nucleus, which is determined through momentum conservation. As discussed in Ref. [12], the distribution of $Q$ values reflects the excitation of the targetlike nucleus. The $E_{\text {rel }}$ distribution is determined using the expression

$$
E_{\mathrm{rel}}=\frac{m_{2} E_{1}+m_{1} E_{2}-2 \sqrt{m_{1} E_{1} m_{2} E_{2}} \cos \theta_{12}}{m_{1}+m_{2}},
$$

where $\theta_{12}$ is the measured laboratory frame opening angle, given by

$$
\cos \theta_{12}=\cos \theta_{1} \cos \theta_{2}+\sin \theta_{1} \sin \theta_{2} \cos \left(\phi_{1}-\phi_{2}\right),
$$




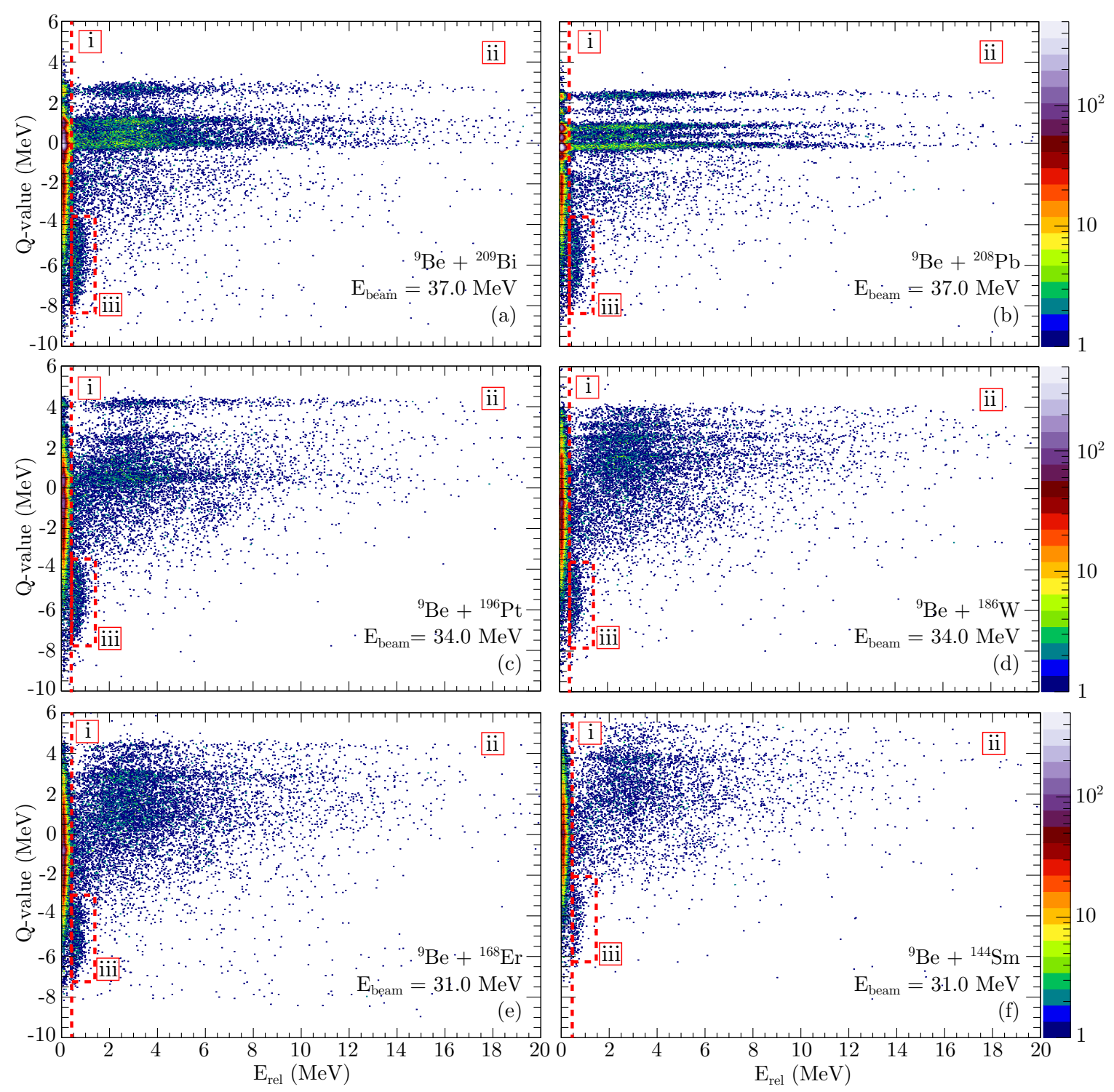

FIG. 1. Spectra of the reconstructed $E_{\text {rel }}$ against $Q$ value for the reactions studied in this reanalysis. Measurements at center-of-mass energies of $E_{\text {c.m. }} / V_{B} \sim 0.9$ are shown. Events arising from the breakup of ${ }^{8} \mathrm{Be}$ from its $0^{+}$ground state, which includes contributions from direct $\left({ }^{9} \mathrm{Be} \rightarrow{ }^{8} \mathrm{Be}_{0^{+}}+n\right)$ and transfer-triggered breakup are shown to the left of the vertical dashed line denoted region (i). Events from breakup of ${ }^{8} \mathrm{Be}$ from either the high excitation energy tail of the $0^{+}$state or the $2^{+}, 4^{+}$states lie to the right of the line [region (ii)], except those marked by the dashed box (iii), which contains direct breakup events from the decay of ${ }^{9} \mathrm{Be}$ from its $\frac{5}{2}^{-}$state.

$m_{i}$ and $E_{i}$ are the mass and energy of each fragment, and $\left(\theta_{i}, \phi_{i}\right)$ is the measured scattering angle and azimuthal angle of each signal in a coincidence event. The $E_{\text {rel }}$ distribution reflects the excitation of the projectilelike nucleus, modified by postbreakup Coulomb interactions of the fragments with the targetlike nucleus. Events with small relative energy $E_{\text {rel }} \lesssim$ $180 \mathrm{keV}$, labeled region (i), result from breakup of the ${ }^{8} \mathrm{Be}$ ground state with $E_{\text {rel }}=92 \mathrm{keV}$. The spread in measured $E_{\text {rel }}$ up to $180 \mathrm{keV}$ results from the angular size of the detector pixels [20]. This unbound state has a width $\Gamma=5.57 \pm 0.25 \mathrm{eV}$, and therefore mean life $\tau=1.2 \times 10^{-16} \mathrm{~s}$ [14]. Owing to this long mean life, breakup will occur asymptotically far from the targetlike nucleus, such that the gradient of the Coulomb field accelerates the two fragments in essentially the same direction.
However, events with large $E_{\text {rel }}$, labeled as region (ii), is associated with the breakup of ${ }^{8} \mathrm{Be}$ resulting in high relative energy. One contributor to such events is the breakup of ${ }^{8} \mathrm{Be}$ from its $2^{+}$resonant state. This state has a large width, $\Gamma=1513 \pm 15 \mathrm{keV}$ and thus a mean life $4.35 \times 10^{-22} \mathrm{~s}$ [14]. ${ }^{8} \mathrm{Be}$ populated in this state will therefore break up close to the targetlike nucleus, where fragment-target interactions significantly affect the trajectory of the breakup fragments. It is these events that may influence complete and incomplete fusion cross sections at above-barrier energies.

Events resulting from direct breakup of ${ }^{9} \mathrm{Be}\left({ }^{9} \mathrm{Be}^{*} \rightarrow\right.$ $\alpha+\alpha+n)$ from the $2.43 \mathrm{MeV}^{\frac{5}{2}}$ state [14] are grouped in the region labeled (iii) in each panel. The spread in $Q$ values reflects the fact that the energy carried by the neutron is 


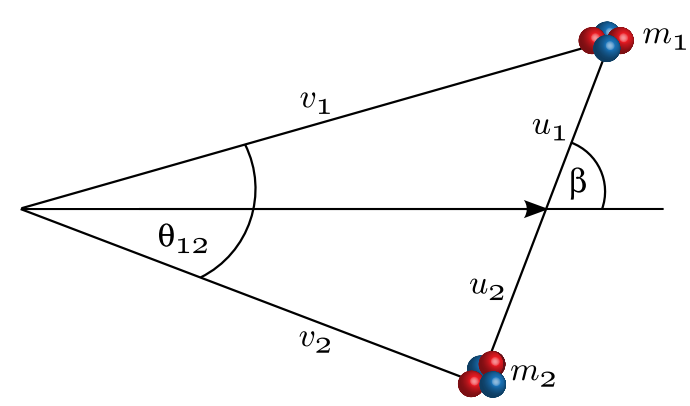

FIG. 2. Diagram demonstrating the relationship between opening angle $\theta_{12}$ and the orientation of the relative momenta of the breakup fragments $\beta . v_{i}$ is the laboratory velocity for each fragment with mass $m_{i}$ and is deduced from their measured energy $E_{i} . u_{i}$ is the velocity of each fragment in their center-of-mass frame, deduced from momentum conservation and their relative energy.

not captured by the BALiN array, resulting in an incorrect reconstruction of the $Q$ value of this breakup mode. Despite missing the neutron, the distribution is relatively sharply peaked in $E_{\text {rel }}(\sim 0.6 \mathrm{MeV})$, reflecting the long mean life of $\tau \sim 8.4 \times 10^{-19} \mathrm{~s}(\Gamma=0.78 \pm 0.13 \mathrm{keV})$ of this state [14]. Breakup from this state will thus also occur far from the targetlike nucleus, giving minimal differential acceleration of the $\alpha$ particles following breakup.

Independent of expectations based on the known mean lives of resonant states, deduced from their widths, it is possible to experimentally separate breakup close to the target nucleus from breakup (asymptotically) far away by examining the energy and angular correlations of the resulting fragments [17,21]. When breakup occurs asymptotically, which is also associated with a well-defined excitation energy $E_{x}$ of the projectilelike nucleus, the laboratory opening angle between the two fragments, $\theta_{12}$, and the orientation of the relative momentum of the breakup fragments, $\beta$, in their center-of-mass frame are related. These quantities, $\theta_{12}$ and $\beta$, are shown in Fig. 2, which can be used to obtain the relationship

$$
\sin \beta=\frac{v_{1} v_{2} \sin \theta_{12}}{\left(v_{2}^{2} u_{1}^{2}+v_{1}^{2} u_{2}^{2}+2 u_{1} u_{2} v_{1} v_{2} \cos \theta_{12}\right)^{1 / 2}} .
$$

Here $v_{i}$ is the laboratory velocity for each fragment, deduced from their measured energy $E_{i}$, and $u_{i}$ is the velocity of each fragment in their center-of-mass frame, deduced from momentum conservation and their relative energy $E_{\text {rel }}=$ $\frac{1}{2} \mu_{12}\left(u_{1}+u_{2}\right)^{2}, \mu_{12}=\frac{m_{1} m_{2}}{m_{1}+m_{2}}$. The $\theta_{12}-\beta$ distributions, reconstructed from the measured data for ${ }^{9} \mathrm{Be}+{ }^{186} \mathrm{~W}$ at $E_{\text {beam }}=$ $37.0 \mathrm{MeV}$, are shown in Fig. 3 for $Q>-3 \mathrm{MeV}$ (panel a), where transfer-triggered breakup is dominant, and for $Q<-3 \mathrm{MeV}$ (panel b). The latter includes contributions from direct breakup, which are those shown in region (iii) of Fig. 1(d) for the same system at $E_{\text {beam }}=34.0 \mathrm{MeV}$. The lines overlaid on the data in Fig. 3 correspond to calculations using Eq. (4) for $E_{x}$ corresponding to breakup from (from left to right) ${ }^{8} \mathrm{Be} 0^{+}, E_{x}=92 \mathrm{keV} ;{ }^{9} \mathrm{Be} \frac{5}{2}^{-}, E_{x}=600 \mathrm{keV}$ [region (iii) in Fig. 1]; and ${ }^{8} \mathrm{Be} 2^{+}, E_{x}=3.03 \mathrm{MeV}$. As can be seen in the figure, bands with excellent correspondence to the

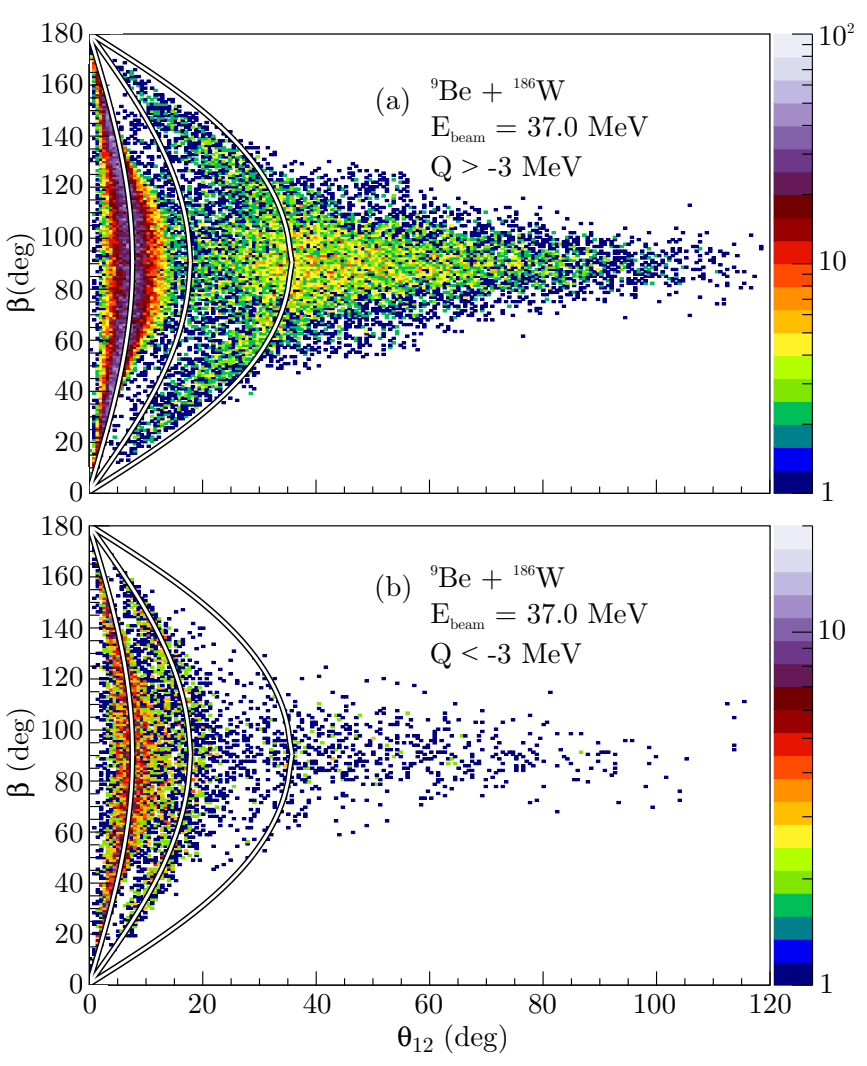

FIG. 3. Deduced experimental $\theta_{12}-\beta$ distribution for the breakup of ${ }^{8} \mathrm{Be}$ formed after neutron transfer from ${ }^{9} \mathrm{Be}$ in interactions with ${ }^{186} \mathrm{~W}$ at $E_{\text {beam }}=37 \mathrm{MeV}$. Panel (a) shows $Q>-3 \mathrm{MeV}$ to highlight transfer-triggered breakup, and (b) shows events with $Q<-3 \mathrm{MeV}$, where the direct ${ }^{9} \mathrm{Be} \frac{5}{2}^{-}$curve is more clearly seen. Lines indicate $\theta_{12}-\beta$ curves calculated for the asymptotic breakup of (left to right) ${ }^{8} \mathrm{Be} 0^{+},{ }^{9} \mathrm{Be} \frac{5}{2}^{-}$, and ${ }^{8} \mathrm{Be} 2^{+}$. Distributions that deviate from these curves are a result of breakup that occurs sufficiently close to the targetlike nucleus to perturb the final trajectories of the breakup fragments. If particles fall into the same pixel of BALiN, they do not register as coincidence events, resulting in a reduced number of events observed near $\beta=0^{\circ}$ and $180^{\circ}$.

calculations for the asymptotic breakup of ${ }^{8} \mathrm{Be} 0^{+}$and ${ }^{9} \mathrm{Be} \frac{5}{2}^{-}$ are present in the experimental $\theta_{12}-\beta$ distribution, confirming the interpretation that these events correspond to breakup asymptotically far from the targetlike nucleus. However, as can be seen in Fig. 3(a), the calculation assuming asymptotic breakup of ${ }^{8} \mathrm{Be} 2^{+}$does not match the data well. This can be explained as a result of breakup occurring close to the targetlike nucleus. When this occurs, the initial kinetic energy of the fragments is small, and their energies are stored in the fragment-target potential. As a result, there is an increased probability for $E_{1} \sim E_{2}$ and thus of deduced values of $\beta \sim 90^{\circ}$ for breakup into identical fragments [17]. Therefore, without making any assumption of the state that is populated, the concentration of events around $\beta \sim 90^{\circ}$ indicates breakup close to the target nucleus. Thus, it is these events that may influence complete fusion cross sections. The extraction of breakup probabilities for these events is the subject of Secs. III and $\mathrm{V}$. 


\section{B. Cross-section normalization}

To extract breakup probabilities, the array was partitioned into $5^{\circ}$ bins covering laboratory angles from $\theta=130^{\circ}$ to $165^{\circ}$. The yield of breakup events in each bin must be normalized to the yield of Rutherford scattering. Elastic events for normalization were extracted from a $\theta$ bin of the BALiN array from $124^{\circ}-127^{\circ}$, where the elastic yield is pure Rutherford for deep sub-barrier measurements. At higher energies, the yield was corrected by up to $11 \%$, determined from optical model calculations, described in Appendix B. Recent precision measurements of the spatial positioning of the BALiN detectors have resulted in slight changes in the location of the array relative to the beam axis. This has resulted a $9 \% \pm 1 \%$ decrease in the number of elastic particles assigned to the $124^{\circ}-127^{\circ}$ bin for each measurement compared to those reported in Ref. [12].

\section{IMPROVED METHOD FOR COINCIDENCE EFFICIENCY DETERMINATION}

To determine absolute breakup probabilities, the coincidence efficiency $\epsilon$ of BALiN had to be determined. In this work, a new two-step approach to efficiency determination was implemented with minimal reliance on simulated breakup distributions. The first step was to calculate the geometric coincidence efficiency of the BALiN array as a function of $\theta_{12}$ and breakup pseudoangle $\theta_{{ }^{8} \mathrm{Be}}$ (described below). A Monte Carlo simulation was used to obtain these geometric coincidence efficiencies, using the breakup code PLATYPUS. Under the assumption of isotropic breakup, the efficiencies derived in this step were model independent. However, the geometric coincidence efficiencies did not account for the events that fall outside of the detector acceptance in $\left(\theta_{8} \mathrm{Be}\right.$, $\left.\theta_{12}\right)$. The second step in the efficiency determination was to simulate the total distribution of fragments to correct for those events with $\theta_{12}$ that fall outside the detector acceptance for each $\theta_{8} \mathrm{Be}$. This correction was small; the events comprised $\sim 7 \%$ of the total yield in the $\theta_{8} \mathrm{Be}$ acceptance of the detector. These simulations were done using a version of PLATYPUS which incorporated the modifications discussed in Sec. IV. Full details of the efficiency determination is described in Appendix $\mathrm{C}$, and a comparison of the efficiencies calculated in this work to those of Ref. [12] is presented in Appendix D.

The breakup pseudoangle is also needed to extract breakup functions from coincidence data. When a reaction produces only one nucleus related to the lighter collision partner in the outgoing trajectory, the angular distribution and distance of closest approach of the projectile and target nuclei may be estimated from the measured scattering angle in a straightforward manner. In a breakup reaction producing pairs of particles which will have different angles $\theta$ and $\phi$, an appropriate way to extract the breakup function is by use of $\theta^{8} \mathrm{Be}$, which can be interpreted as the reconstructed scattering angle of the ${ }^{8} \mathrm{Be}$ had it not broken up. This is related to the deduced recoil angle of the targetlike nucleus $\theta_{\text {recoil }}$. The latter is already used to calculate the kinetic energy of the recoiling targetlike nucleus and thus the $Q$ value of the breakup reactions. $\theta_{\text {recoil }}$ is determined from the momenta of the measured breakup fragments using momentum conservation, and $\theta_{8}{ }_{\mathrm{Be}}$ is given by

$$
\tan \theta_{\mathrm{Be}}=\frac{\sin 2 \theta_{\text {recoil }}}{M_{p} / M_{t}-\cos 2 \theta_{\text {recoil }}},
$$

where $M_{p}$ is the mass of the projectilelike nucleus and $M_{t}$ the mass of the targetlike nucleus.

\section{CLASSICAL TRAJECTORY SIMULATIONS}

The ultimate aim of this work is to understand the contribution that transfer-triggered breakup makes to the suppression of complete fusion at energies above the barrier. By making below-barrier measurements of no-capture breakup probabilities and relating these probabilities to above-barrier $\mathrm{CF}$ and ICF cross sections, it is possible to determine the contributions of breakup to suppression of CF and to cross sections for ICF products. However, to achieve this, a reliable simulation of postbreakup trajectories of the fragments is required. This is for two reasons: first, to extract the belowbarrier near-target breakup probabilities from experimental results, and second, to take these experimentally determined breakup probabilities and make predictions of CF and ICF at above-barrier energies.

As no fully quantum mechanical model of transfer-induced breakup exists yet, classical simulations have been performed. Clearly, it is important that a classical model captures the key physics of the breakup processes. Namely, (a) the locations of the transfer reactions, (b) the properties of the intermediate nucleus populated after transfer, and (c) the subsequent decay and postbreakup acceleration of the fragments. The acceleration of the fragments after breakup has the capacity to change their relative energy and is the classical analog of continuum-continuum couplings in quantum mechanical models. The classical dynamical breakup code PLATYPUS $[15,16]$, with modifications described below, provides an appropriate platform for these calculations. PLATYPUS is a three-body classical trajectory model with stochastic breakup that enables calculations of breakup observables as well as incomplete and complete fusion cross sections. It considers a target and a weakly bound pseudoprojectile (here, ${ }^{8} \mathrm{Be}$ ) that initially follow Rutherford trajectories. Breakup probabilities and locations are stochastically sampled from an experimentally determined breakup function $P\left(R_{\min }\right)$. At the point of breakup, the properties of the fragments (excitation energy $E_{x}$, separation, orientation) are stochastically sampled before propagating in the fragment-fragment and fragment-target fields. Several significant modifications to PLATYPUS have been made to more accurately capture the details of breakup dynamics, as described below.

\section{A. Incorporating excitation energies and lifetimes of resonant states of the projectilelike nucleus}

To include the known low-energy structure of ${ }^{8} \mathrm{Be}$, modifications to PLATYPUS were made to model the resonant states in ${ }^{8} \mathrm{Be}$. The energy and angular distribution of breakup fragments produced after the decay of a projectilelike nucleus populated in transfer reactions depends critically on (i) the excitation of the projectilelike nucleus that breaks up and (ii) the location 
of breakup with respect to the targetlike nucleus, which is, in turn, sensitive to the lifetime of the projectilelike nucleus after the point of transfer. In the previous versions of PLATYPUS, the excitation of the projectilelike nucleus was given as a range from $E_{\min }$ to $E_{\max }$ with either a flat or exponentially decreasing distribution [16]. Although lifetimes were not treated explicitly, breakup fragments would take some time to propagate from their assumed initial Gaussian distribution of separations to beyond their mutual barrier radius [15]. This effective lifetime is sensitive to the fragment-fragment potential.

As demonstrated below, the population of ${ }^{8} \mathrm{Be}$ in the reactions studied in this work can be well described as a combination of $0^{+}$ground state and first excited $2^{+}$state. Thus, the simulated excitation energy and lifetime distributions of ${ }^{8} \mathrm{Be}$ should correspond to the width of these states. Modifications to PLATYPUS were made such that excitation energies sampled from realistic distributions of excitation energy have a corresponding mean life associated with each excitation energy. The excitation energy probability distributions were calculated from the one-state, one-channel limit of $R$-matrix theory [18,19]. The corresponding mean life was estimated using $\tau\left(E_{x}\right)=\hbar / \Gamma_{\ell}\left(E_{x}\right)$, where $\Gamma_{\ell}\left(E_{x}\right)$ is the energy-dependent resonance width. This has been recently described in Ref. [22], where excitation energy probability distributions were calculated for ${ }^{6,7} \mathrm{Li}$. Shown in Fig. 4 are the resulting excitation energy probability distributions (a) and
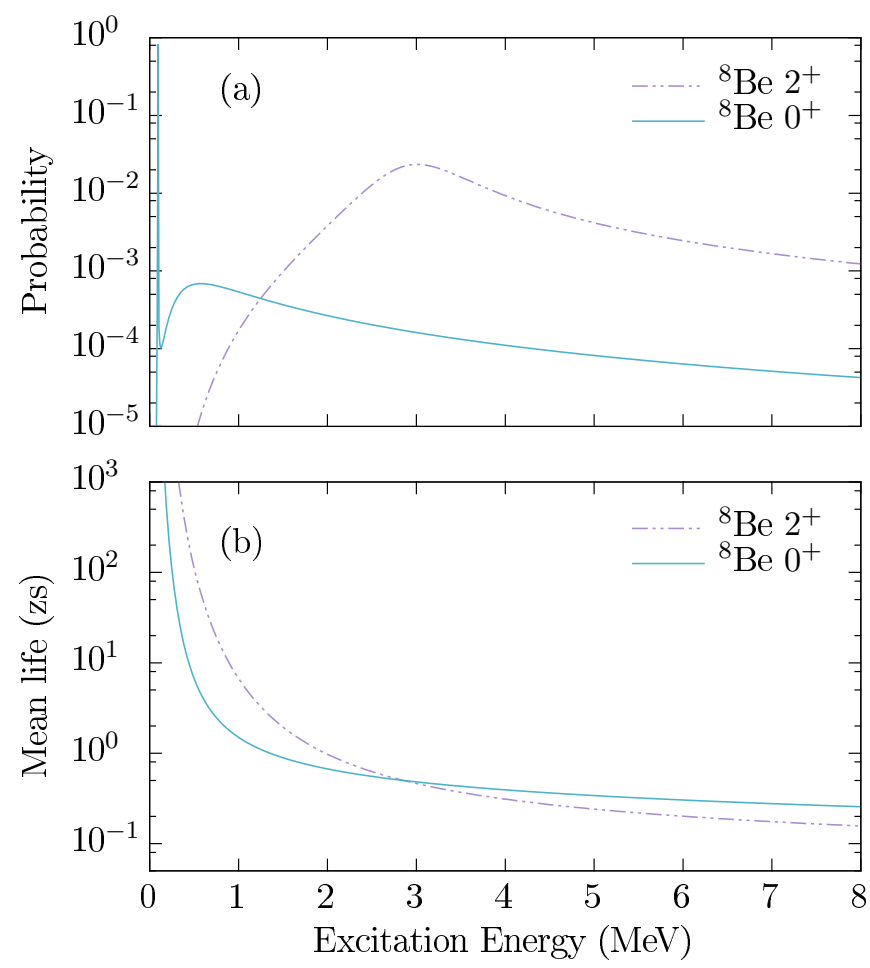

FIG. 4. Excitation energy probability distribution (a) and excitation energy dependant mean life (b) for $0^{+}$(solid line) and $2^{+}$(dashed line) states in ${ }^{8} \mathrm{Be}$ used as input in the modified version of PLATYPUS that explicitly takes into account resonance excitation energies and lifetimes. excitation energy dependent mean lives (b) for the ${ }^{8} \mathrm{Be} 0^{+}$and $2^{+}$states used in the PLATYPUS calculations in this work.

Including these probability distributions of excitation energy and associated mean-life, the distribution of decay (breakup) times of short-lived resonance states are now modelled explicitly in PLATYPUS. The first step is randomly choosing a "transfer radius," $R_{\mathrm{Tr}}$, according to the breakup function as originally done. Then a classically allowed excitation energy $E_{x}$ (with corresponding mean life $\tau$ ) is chosen from the distribution of excitation energies as shown in Fig. 4. The projectile then propagates along its trajectory for some time $t$, sampled from the exponential distribution of times expected from the mean life, $e^{-t / \tau}$, before breaking up into two fragments with relative energy corresponding to $E_{x}$. The fragments are initially placed at a separation radius corresponding to the peak of their mutual barrier. Breakup is thus defined to occur when the two fragments pass their mutual barrier. Crucially, ${ }^{8}$ Be produced by transfer before the distance of closest approach may pass the turning point and begin to recede from the target before breaking up.

This explicit handling of excitation energies and mean lives gives a more physically realistic (though still phenomenological) distribution of (i) breakup fragment energy and (ii) the time taken between transfer and breakup, and thus positions along the trajectories. The latter modification in particular removes sensitivity to the fragment-fragment potential. In addition, these modifications allow long-lived states, such as the ${ }^{8} \mathrm{Be}$ ground state, to be simulated with PLATYPUS rather than requiring an additional simulation with a different code [12]. Further, requiring that the distribution of excitation energies used in PLATYPUS be determined by the known resonance properties of ${ }^{8} \mathrm{Be}$ removes this quantity as a parameter in the model and, as discussed in Sec. VI, has a significant effect on $\mathrm{CF}$ and ICF predictions.

\section{B. Incorporating effects of excitation of targetlike nuclei}

As can be seen by the spread of $Q$ values in Fig. 1, the targetlike nucleus is populated with a large range of excitations (up to $\sim 8 \mathrm{MeV}$ ) in these reactions. Trivially, as the excitation energy of the targetlike nucleus increases, the energy available for the excitation of the projectilelike nucleus decreases. This results in a decrease in $E_{\text {rel }}$ (as can also be seen in Fig. 1) and thus a decrease in average opening angle $\theta_{12}$. Therefore, the fidelity of the reproduction of experimental results in PLATYPUS is also dependent on the distribution of targetlike excitations.

PLATYPUS, being a classical model, has radii around the classical turning point where transfer is classically forbidden owing to energy conservation. The size of this region depends on the beam energy, angular momentum, and the excitations of the projectilelike and targetlike nuclei. The latter was not incorporated in the original version of PLATYPUS, which was thus modified to include the excitation energy distribution of the targetlike nucleus, obtained from the experimentally determined $Q$-value distribution. As a result, the PLATYPUS simulation now reflects both the excited states of the targetlike nucleus and the probability of populating those states in the neutron transfer reactions studied in this work. To model the excitation energy, at $R_{\operatorname{Tr}}$ an equivalent amount of kinetic 
energy is deducted from the projectilelike nucleus such that the direction of the relative velocity of the system is maintained.

\section{Modifications to the local breakup function}

The aim of these below-barrier measurements of breakup is to determine the breakup probabilities $P$ as a function of $R_{\min }$, the distance of closest approach on a Coulomb trajectory. The experimental data were fitted with the functional form

$$
P\left(R_{\min }\right)=e^{\mu R_{\min }+v},
$$

where $\mu$ and $v$ are the (logarithmic) slope and intercept of the function, respectively. This function is interpreted as the integral of the local reaction probability $\mathcal{P}(R)$ along the classical orbit of the projectile,

$$
P\left(R_{\min }\right)=2 \int_{R_{\min }}^{\infty} \mathcal{P}(R) d R .
$$

$\mathcal{P}(R)$ is a function of the projectile-target separation $R$, and $\mathcal{P}(R) d R$ gives the reaction probability between $R$ and $R+d R$. The factor of two reflects the initial assumption that, taking breakup to be instantaneous, it can occur with equal probability on the ingoing and outgoing trajectories. With the incorporation of resonance lifetimes, the local probability must now be interpreted as that for the trigger event for breakup, in this case transfer. At above-barrier energies, when using PLATYPUS to estimate $\sigma_{\mathrm{ICF}}$, the distance of closest approach is inside the barrier radius; thus, only the transfer probabilities on the ingoing trajectory should included. This change by a factor of two has been taken into account in the modified PLATYPUS calculations of $\sigma_{\mathrm{ICF}}$, resulting in a decrease in contributions to $\sigma_{\text {ICF }}$ from trajectories with angles within the grazing angle by approximately a factor of two.

The distribution of transfer positions along the projectiletarget trajectory has also been modified. In the original PLATYPUS, when determining the probability along the trajectory, it is assumed that because

$$
2 \int_{R_{\min }}^{\infty} \mathcal{P}(R) d R=e^{\mu R_{\min }+v},
$$

the local probability must then have the form [15]:

$$
\mathcal{P}(R) \propto e^{\mu R} .
$$

However, this neglects the fact that interacting nuclei spend more time near the distance of closest approach than at other distances. As a result, $d P\left(R_{\min }\right) / d t$ goes to zero at the point of closest approach, as illustrated in Appendix E for a classical Coulomb trajectory.

Instead, we assign each time step on a particular projectile trajectory a relative probability assuming a local (transfer) probability $\widetilde{\mathcal{P}}(t) \propto e^{\mu R(t)}$ and normalize the full trajectory such that

$$
P\left(R_{\min }\right)=\int_{-\infty}^{\infty} \widetilde{\mathcal{P}}(t) d t .
$$

The local probability is then peaked at the distance of closest approach, which is physically more reasonable.

\section{Comparison with experimental data}

The accuracy of the PLATYPUS simulations was assessed by comparing them with the experimentally measured $\theta_{12}-\beta$ distributions. The $\theta_{12}-\beta$ distributions are a good test because they are sensitive to the effect of fragment-target interactions and therefore to the position and energetics of breakup [17]. The experimental $\theta_{12}-\beta$ distribution for the breakup of ${ }^{8} \mathrm{Be}$ formed following neutron transfer in collisions of ${ }^{9} \mathrm{Be}$ with ${ }^{209} \mathrm{Bi}$ is shown in Fig. 5(a). It is compared with modified and unmodified PLATYPUS simulations in Figs. 5(b) and 5(c), respectively. As the original PLATYPUS does not simulate longlived states, the $0^{+}$state seen in the intense purple band at small $\theta_{12}$ in Fig. 5(a) has not been included. In the modified PLATYPUS simulation, both $0^{+}$and $2^{+}$resonances have been simulated, and the distributions are combined to produce the same ratio of breakup events that populate the $E_{\text {rel }}=92 \mathrm{keV}^{+}$peak to the total number of events, as seen in the experimental data.

As discussed in Sec. II, the effect of fragment-target Coulomb interactions results in deviations in the $\theta_{12}-\beta$ distribution from that expected for asymptotic breakup [calculated using Eq. (4)]. The modified version of PLATYPUS well reproduces the $0^{+}$peak and reproduces the high $\theta_{12}$ component better than the unmodified model (in particular, events below the diagonal red dashed line, which is drawn to guide the eye). However, the simulation contains a higher intensity of events with $\theta_{12} \gtrsim 60^{\circ}$ and $\beta \sim 90^{\circ}$. This means that too many breakup events result in coincident fragments with similar
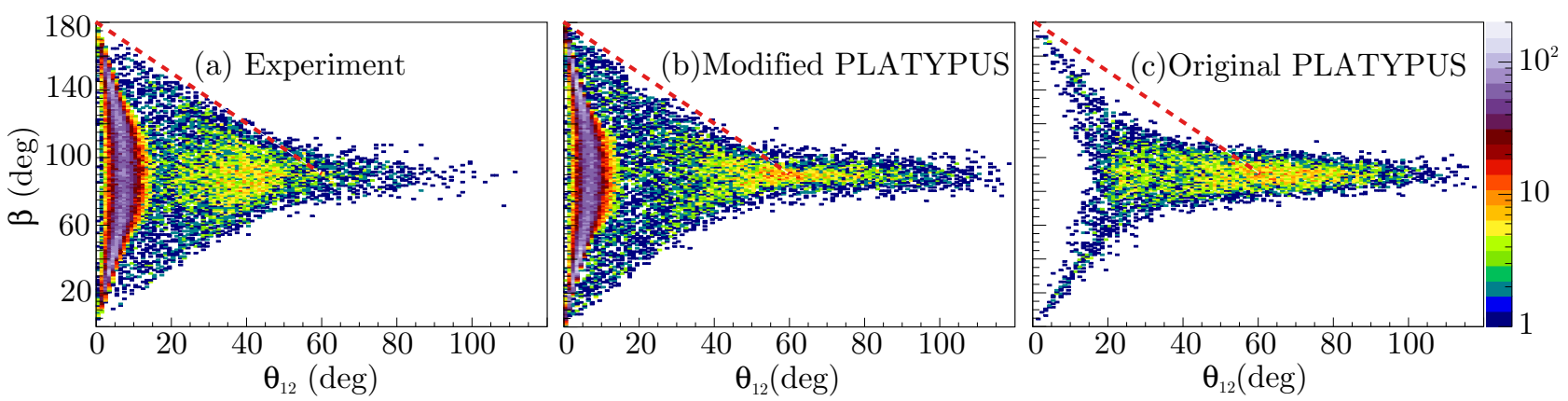

FIG. 5. (a) Measured $\theta_{12}-\beta$ distribution for the breakup of ${ }^{8} \mathrm{Be}$ formed following neutron transfer in interactions of ${ }^{9} \mathrm{Be}$ with ${ }^{209} \mathrm{Bi}$ at $E_{\text {beam }}=34.0 \mathrm{MeV}$. (b) The corresponding modified PLATYPUS simulation, which includes contribution from ${ }^{8} \mathrm{Be} 0^{+}$and $2^{+}$resonances. (c) The corresponding unmodified PLATYPUS simulation, with $0.95 \leqslant E_{x} \leqslant 4 \mathrm{MeV}$, approximating the ${ }^{8} \mathrm{Be} 2^{+}$resonance only. The red diagonal line provides a reference to quantify the differences between the observables for the $2^{+}$resonance. 
energies and large opening angles. This discrepancy could be ameliorated by considering the effect of the projectiletarget potential in producing a preferential orientation for ${ }^{8} \mathrm{Be}$ relative to the target, as has been previously explored for the direct breakup of ${ }^{7} \mathrm{Li}$ [23]. However, without a satisfactory method for reliably parametrizing orientation effects, they are neglected, and all breakup is assumed to occur isotropically in the rest frame of ${ }^{8} \mathrm{Be}$. Nevertheless, these simulations demonstrate that the population of ${ }^{8} \mathrm{Be}$ in the reactions studied in this work can be reasonably well described as a combination of $0^{+}$ground state and $2^{+}$first excited state. Further, the modifications to (i) better model the projectilelike nucleus in resonant states with explicitly included mean lives, (ii) model reactions that result in excitation of the targetlike nucleus, and (iii) better distribute the transfer probability along the projectile-target trajectory provide a more physically realistic, though still phenomenological, model of breakup following transfer.

\section{NEAR-TARGET BREAKUP PROBABILITIES}

The breakup probability is defined for each $\theta_{8} \mathrm{Be} \sim 5^{\circ}$ bin as the ratio between the breakup cross section, determined from the yield of breakup fragments with the reconstructed angle of the unbroken projectile falling in $\theta^{8} \mathrm{Be}$, and the Rutherford scattering cross section for each $\theta_{8} \mathrm{Be}$ bin,

$$
P\left(\theta_{{ }^{8} \mathrm{Be}}\right)=\frac{\left(\frac{d \sigma}{d \Omega}\right)_{\mathrm{BU}}\left(\theta_{{ }^{8} \mathrm{Be}}\right)}{\left(\frac{d \sigma}{d \Omega}\right)_{\mathrm{Ruth}}\left(\theta_{{ }^{8} \mathrm{Be}}\right)} .
$$

The breakup pseudoangle maps to a distance of closest approach of the target and unbroken projectile $R_{\text {min }}$, neglecting the nuclear potential at these sub-barrier energies, according to

$$
R_{\min }=\frac{Z_{1} Z_{2} e^{2}}{2 E_{\mathrm{c} . \mathrm{m} .}}\left(1+\frac{1}{\sin \frac{\theta_{8_{\mathrm{Be}}}}{2}}\right) .
$$

This definition of $R_{\text {min }}$ implicitly assumes that the reconstructed scattering angle of the unbroken projectilelike nucleus is close to the Rutherford angle of the incoming projectile, that is, $\theta_{8_{\mathrm{Be}}} \approx \theta_{\mathrm{Ruth}}$. This assumption can be tested using PLATYPUS simulations. Shown in Fig. 6 is the Rutherford scattering angle of the pseudoprojectile derived from the incident trajectory, $\theta_{\text {Ruth }}$, plotted against the reconstructed breakup pseudoangle, for ${ }^{8} \mathrm{Be}_{2^{+}}+{ }^{207} \mathrm{~Pb} \rightarrow \alpha+\alpha$ at $E_{\text {beam }}=34.0 \mathrm{MeV}$. In the determination of the breakup functions, discussed in Sec. V, these deviations were treated as a correction to $\theta_{8_{\mathrm{Be}}}$, and for each $\theta^{8} \mathrm{Be}$ bin, the average discrepancy between the Rutherford and reconstructed angles was subtracted from $\theta_{8} \mathrm{Be}$. This correction was larger for breakup that occurs close to the targetlike nucleus, and was $Z$ dependent, varying from $\sim 1^{\circ}$ for ${ }^{9} \mathrm{Be}+{ }^{144} \mathrm{Sm}$, to $\sim 6^{\circ}$ for ${ }^{9} \mathrm{Be}+{ }^{209} \mathrm{Bi}$. As such, these discrepancies are likely to result from trajectories that are perturbed by proximity to the high- $Z$ targetlike nucleus.

With the corrected angle $\theta_{8} \mathrm{Be}$ transformed to $R_{\text {min }}$, breakup functions may be determined experimentally from the ratio of efficiency-corrected breakup yield to the elastic yield in each

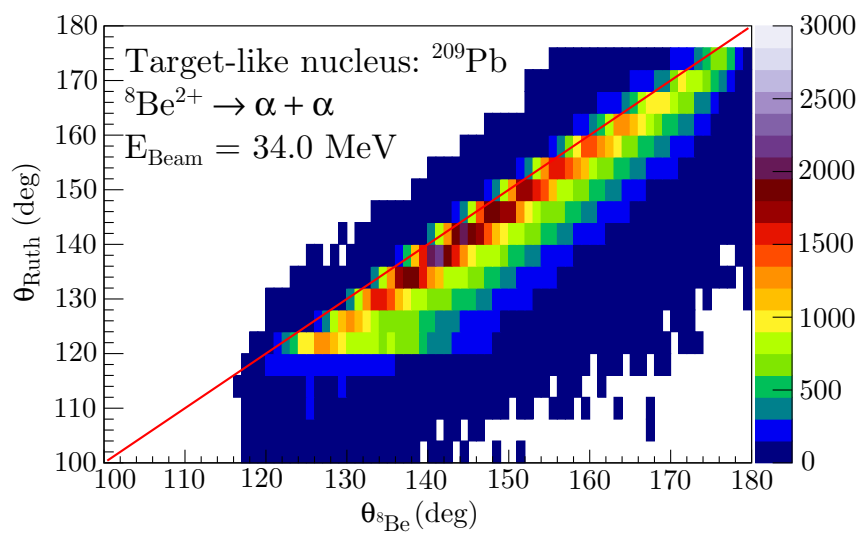

FIG. 6. PLATYPUS simulation for ${ }^{8} \mathrm{Be}_{2^{+}}+{ }^{209} \mathrm{~Pb} \rightarrow \alpha+\alpha+{ }^{209} \mathrm{~Pb}$ at $E_{\text {beam }}=34.0 \mathrm{MeV}$ for events that are captured by BALiN, demonstrating the relatively small difference between the Rutherford scattering angle of the ${ }^{8} \mathrm{Be}$ pseudoprojectile $\theta_{\text {Ruth }}$ and the angle $\theta_{8_{\mathrm{Be}}}$ that is reconstructed from the captured $\alpha$ particles.

$\theta_{8 \mathrm{Be}}$ bin:

$$
P\left(\theta_{8_{\mathrm{Be}}}\right)=\frac{N_{\mathrm{BU}}\left(\theta_{{ }^{8} \mathrm{Be}}\right)}{N_{\text {Ruth }}\left(\theta_{\text {Ruth }}\right)} .
$$

Here $N_{\mathrm{BU}}\left(\theta_{{ }^{8} \mathrm{Be}}\right)$ is the yield of near-target breakup events corrected for efficiency $\epsilon\left(\theta_{12}, \theta^{{ }^{B} \mathrm{Be}}\right)$ and $N_{\text {Ruth }}\left(\theta_{\text {Ruth }}\right)$ the calculated Rutherford yield in a given $\theta_{\text {Ruth }}$ bin. Details of the determination of $N_{\text {Ruth }}\left(\theta_{\text {Ruth }}\right)$ are given in Appendix B.

The resulting probabilities of near-target breakup are shown in Fig. 7(a). Each group of points in $R_{\text {min }}$ represent measurements in $5^{\circ} \theta_{8 \mathrm{Be}}$ bins with different $E_{\text {beam }}$. A least-squares fit using Eq. (6) to the experimental data was performed for each system, indicated by the solid lines in Fig. 7(a). These breakup functions provide a useful comparison to previous work. We also present an alternative parametrization of the breakup function. A perhaps more intuitive way to parametrize breakup probabilities is as a function of the distance of closest approach relative to the average barrier radius $R_{\mathrm{B}}$, in the form of Eq. (4) of Ref. [24], such that

$$
P_{\mathrm{BU}}=P\left(R_{B}\right) e^{\mu\left(R_{\min }-R_{\mathrm{B}}\right)},
$$

where $P\left(R_{B}\right)$ is the probability of breakup along a trajectory that reaches a distance of closest approach $R_{\mathrm{B}}$ and $\mu$ is the same slope parameter as in Eq. (6). A detailed discussion of the physical significance of these parameters can be found in Ref. [24]. $R_{B}$ was parametrized as $R_{B}=1.44\left(A_{T}^{1 / 3}+A_{P}^{1 / 3}\right)$, which reproduced the $R_{B}$ of the calculated São Paulo potentials between the ${ }^{8} \mathrm{Be}$ and the targetlike nucleus within $0.1 \mathrm{fm}$. Where the target nucleus is deformed, as is the case for ${ }^{168} \mathrm{Er}$ and ${ }^{186} \mathrm{~W}$, the breakup function is an average over all orientations. The resulting breakup probabilities are shown in Fig. 7(b). From this, it is apparent that the dependence of breakup probability on the targets studied in this work is fairly small. Instead, near-target breakup is dominantly driven by how close the trajectory comes to $R_{\mathrm{B}}$. This agrees with what was found in Ref. [12].

The fitted breakup slope parameters using both parametrizations are given in Table I. The reported uncertainties $\sigma$ in the 

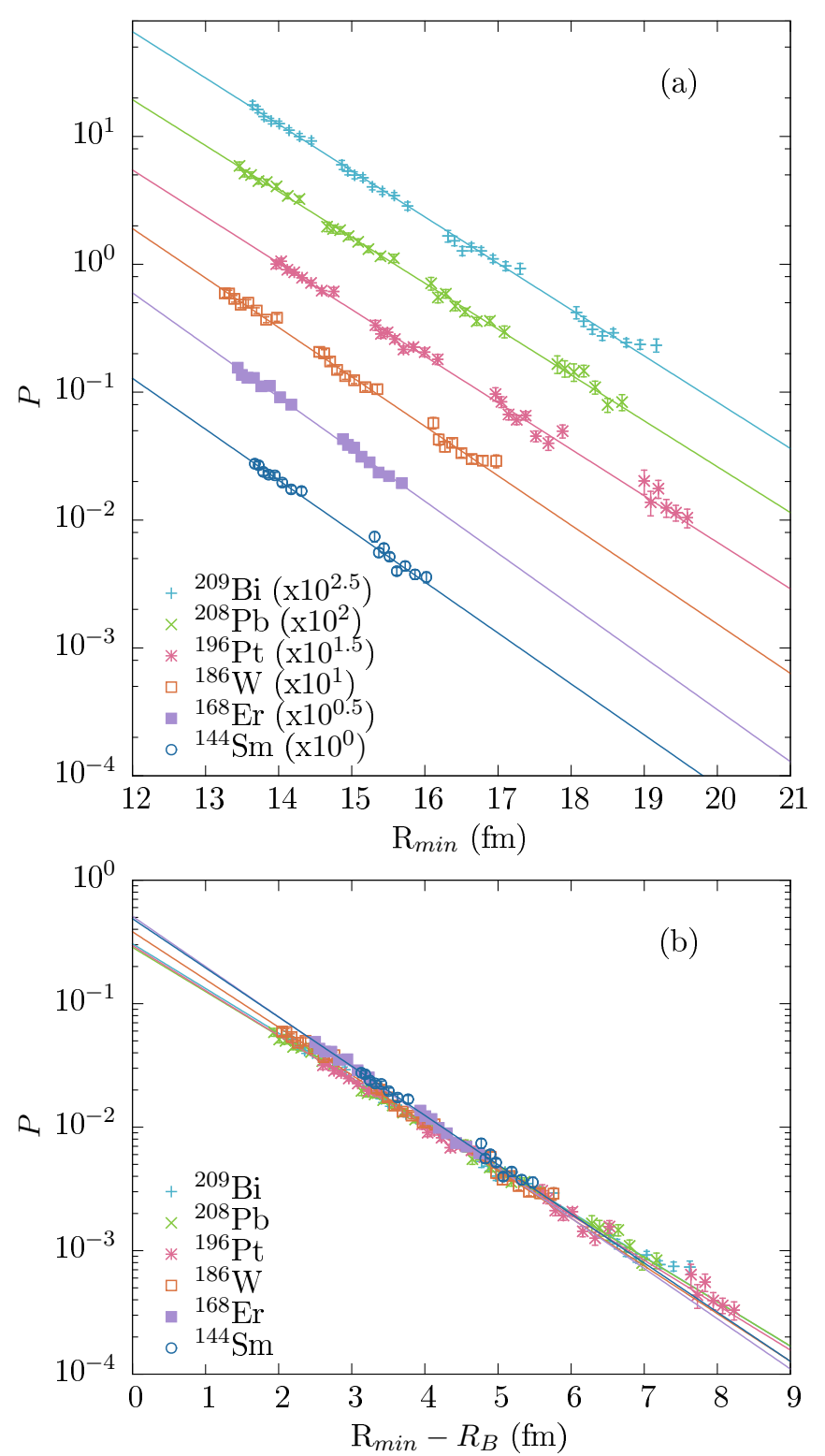

FIG. 7. Measured near-target (region ii of Fig. 1) breakup probabilities for the breakup of ${ }^{8} \mathrm{Be}$ formed following neutron transfer in reactions of ${ }^{9} \mathrm{Be}$ with ${ }^{144} \mathrm{Sm},{ }^{168} \mathrm{Er},{ }^{186} \mathrm{~W},{ }^{196} \mathrm{Pt},{ }^{208} \mathrm{~Pb}$, and ${ }^{209} \mathrm{Bi}$ at energies below the barrier (a) as a function of the separation of the centers of the nuclei, where probability values have been offset for clarity, as indicated in the legend, and (b) as a function of distance from the projectile-target barrier. Lines represent least-squares fits with Eq. (6). Errors in $P$ are statistical and, for the most part, are smaller than the symbol size.

parameters come from each least-squares fit. The parameters $\mu$ and $P\left(R_{B}\right)$ of the breakup functions are shown as a function of $Z_{T}$ in Fig. 8. Unlike those found in Ref. [12], there is a fairly weak $Z_{T}$ dependence on the fitted $\mu$; a line of best fit yields $\mu=0.005 Z_{T}-1.272$. There is also a trend of increasing $P\left(R_{B}\right)$ with decreasing $Z_{T}$. This is correlated with the trend of increasing ground-state neutron stripping $Q$ value with decreasing $Z_{T}$, as well as the number of states available for
TABLE I. Near-target breakup function parameters determined through least-squares fits to the experimental data shown in Fig. 7 for the breakup of ${ }^{8} \mathrm{Be}$ formed after neutron transfer in reactions of ${ }^{9} \mathrm{Be}$ with ${ }^{144} \mathrm{Sm},{ }^{168} \mathrm{Er},{ }^{186} \mathrm{~W},{ }^{196} \mathrm{Pt},{ }^{208} \mathrm{~Pb}$, and ${ }^{209} \mathrm{Bi}$.

\begin{tabular}{lcccccc}
\hline \hline & ${ }^{144} \mathrm{Sm}$ & ${ }^{168} \mathrm{Er}$ & ${ }^{186} \mathrm{~W}$ & ${ }^{196} \mathrm{Pt}$ & ${ }^{208} \mathrm{~Pb}$ & ${ }^{209} \mathrm{Bi}$ \\
\hline$\mu\left(\mathrm{fm}^{-1}\right)$ & -0.92 & -0.94 & -0.89 & -0.84 & -0.83 & -0.83 \\
$\sigma_{\mu}\left(\mathrm{fm}^{-1}\right)$ & 0.02 & 0.02 & 0.01 & 0.01 & 0.01 & 0.01 \\
$\nu$ & 9.0 & 9.6 & 9.0 & 8.3 & 8.3 & 8.4 \\
$\sigma_{v}$ & 0.3 & 0.3 & 0.2 & 0.2 & 0.2 & 0.1 \\
$P\left(R_{B}\right)$ & 0.54 & 0.56 & 0.42 & 0.32 & 0.31 & 0.33 \\
$\sigma_{P\left(R_{B}\right)}$ & 0.05 & 0.03 & 0.02 & 0.02 & 0.01 & 0.01 \\
\hline \hline
\end{tabular}

population near the optimum $Q$ value of $0 \mathrm{MeV}$. It would be interesting to see how these trends evolve as $Z_{T}$ decreases.

While the breakup functions derived in this work are comparable to those found by Rafiei et al. [12], there is an average increase in the probability of breakup by a factor of $1.14 \pm 0.09$ at $R_{\min }-R_{\mathrm{B}}=4 \mathrm{fm}$. These differences result from the combined effects of several factors that have been discussed above, but are summarized here: (i) The Rutherford scattering yield in the normalization bin for every measurement is a factor of $0.921 \pm 0.009$ lower owing to slight refinement in the actual
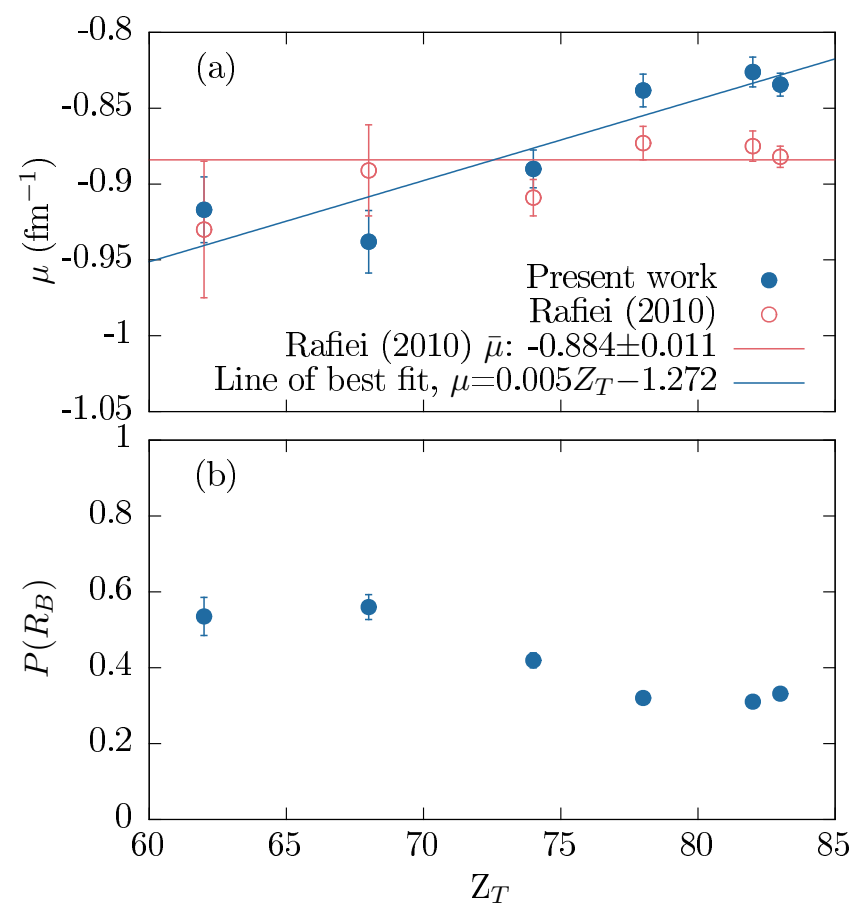

FIG. 8. (a) Solid circles show the slope parameters $\mu\left(\mathrm{fm}^{-1}\right)$ derived from least-squares fits to the experimental data shown in Fig. 7(b), fit with $P_{\mathrm{BU}}=P\left(R_{B}\right) e^{\mu\left(R_{\min }-R_{\mathrm{B}}\right)}$, shown as a function of target, $Z_{T}$. There is a slight $Z_{T}$ dependence on the slope, indicated by the line of best fit $\mu=0.005 Z_{T}-1.272$. Open circles show results from Ref. [12], which have mean slope $\bar{\mu}=-0.884 \pm 0.011$ (red line). The reasons for the discrepancies between the present and previous works are discussed in the text. (b) Corresponding $P\left(R_{B}\right)$ values derived from least-squares fits to the experimental data shown in Fig. 7(b), using Eq. (14). 
position of the BALiN array; (ii) the coincidence efficiency of these $\alpha-\alpha$ pairs calculated using PLATYPUS with respect to $\theta_{12}$ is different from that deduced in the previous work and has a different $Z_{T}$ and $E_{\text {beam }}$ dependence; and (iii) correcting for coincidence efficiency produces an efficiency-corrected yield over all azimuthal angles, and the calculation of the Rutherford yield must reflect this, as discussed in Appendix B. As seen in Fig. 8, the slope, $\mu$, of the breakup function becomes shallower with increasing $Z_{T}$. The difference in average slope from the previous work is primarily driven by the two-dimensional coincidence efficiency correction used in this work. The improvements to PLATYPUS and to the efficiency determinations allow reliable cross sections to be determined, which could be analyzed using available semiclassical methods [25,26]. With these new breakup functions, the next step is then to determine the impact of breakup on fusion suppression with the modified PLATYPUS model.

\section{ABOVE-BARRIER INCOMPLETE FUSION CROSS SECTIONS}

There have been two major approaches towards characterizing fusion suppression in collisions with weakly bound nuclei. The first is through comparing measured above-barrier complete fusion cross sections to coupled-channels predictions of fusion cross sections $\sigma_{\mathrm{CF}}^{\text {expt }} / \sigma_{\text {fus }}^{\text {calc }}$ (e.g., Refs. [1,2,7-9]). This approach relies on accurate determination of the average barrier energy [2] and is somewhat model dependant [27]. The second approach equates fusion suppression to the fraction of incomplete fusion to total fusion $F_{\mathrm{ICF}}=\frac{\sigma_{\mathrm{ICF}}}{\sigma_{\mathrm{ICF}}+\sigma_{\mathrm{CF}}}$. Incomplete fusion is defined experimentally as capture of only part of the charge of the projectile. This approach is justified by measurements which find similar values for $\left(1-\sigma_{\mathrm{CF}}^{\text {expt }} / \sigma_{\text {fus }}^{\text {calc }}\right)$ and $F_{\mathrm{ICF}}[1]$. As such, experimental measures of $F_{\mathrm{ICF}}$ are thought to provide an indirect measure of fusion suppression that is model independent.

When trying to understand the role of breakup in the observed suppressions of complete fusion, it has been conjectured that $\sigma_{\mathrm{ICF}}$ (and thus $F_{\mathrm{ICF}}$ ) is entirely attributable to breakup of the weakly bound nucleus followed by capture of one of the fragments. However, it is very difficult to separate breakup followed by capture of one of the fragments from a transfer process forming the same nucleus. If transfer comprises a large fraction of $\sigma_{\mathrm{ICF}}, F_{\mathrm{ICF}}$ cannot be attributed solely to breakup. Further, $\sigma_{\mathrm{ICF}}+\sigma_{\mathrm{CF}}$ can no longer be interpreted as the total fusion cross section. In the case of ${ }^{7} \mathrm{Li}+{ }^{165} \mathrm{Ho}$, exclusive measurements of $\gamma$ rays and charged fragments favor the interpretation that $\sigma_{\text {ICF }}$ is predominantly attributable to breakup [28]. While the interpretation of $\sigma_{\mathrm{ICF}}$ is ambiguous experimentally, it is clear within a classical model. By using PLATYPUS, the contribution of breakup to $F_{\mathrm{ICF}}$ can be determined.

PLATYPUS is designed to provide predictions of $\sigma_{\mathrm{CF}}$ and $\sigma_{\mathrm{ICF}}$ at energies above the barrier, through the use of the experimentally determined breakup functions, applied at above-barrier energies. In PLATYPUS, ICF is assumed to occur when one of the breakup fragments passes inside the barrier radius, while CF occurs when either the unbroken projectile or both breakup fragments pass the barrier radius. Calculations were performed using the near-target breakup functions determined from the least-squares fit to the below-barrier experimental breakup data, which have parameters as shown in Table I. Nuclear potentials were calculated using the São Paulo potential [29]. Calculations were performed for partial waves up to $100 \hbar$, with 200000 breakup events simulated in total. The yield of neartarget transfer-triggered breakup was attributed exclusively to the breakup of the $2^{+}$resonance in ${ }^{8} \mathrm{Be}$, and thus the modelled excitation energies and lifetimes of the ${ }^{8}$ Be projectile were those of the $2^{+}$state, as shown in Fig. 4. Near-target breakup of ${ }^{8} \mathrm{Be}$, in addition to arising from the $2^{+}$state, should have some contribution from the high excitation energy tail of the $0^{+}$state. Test calculations show that this contribution should be expected to decrease the overall $F_{\mathrm{ICF}}$ arising from near-target transfer-triggered breakup. This is because the average excitation energy of the high-energy tail of the $0^{+}$ state is lower than that of the $2^{+}$state, as can be seen in Fig. 4. Hence, the average lifetime is longer, and a smaller fraction of near-target breakup will occur prior to reaching the fusion barrier. Calculations of $F_{\mathrm{ICF}}$ were made at energies in $0.05 V_{B}$ steps from $1.05 V_{B}-1.30 V_{B}$, consistent with previous work [12]. Over the energy range of $1.05 V_{B}-1.30 V_{B}, F_{\text {ICF }}$ is energy dependent and varies by a factor of two for each reaction, from $\mathrm{F}_{\mathrm{ICF}}=0.16$ at $1.05 V_{B}$ to 0.08 at $1.30 V_{B}$ on average. The results from each energy step have been averaged to give a $F_{\text {ICF }}$ value for each system to allow comparison with previous work and to experimental measures of complete fusion suppression.

The resulting $\sigma_{\mathrm{CF}}$ and $\sigma_{\mathrm{ICF}}$ are presented as $F_{\mathrm{ICF}}$ shown by the solid circles (blue) in Fig. 9. In contrast to expectations from the empirical prediction of Ref. [11], these new predictions show no significant dependence on target $Z$ in the range studied in this work and have a mean value of $0.11 \pm 0.02$, which is indicated by the solid line Fig. 9. For comparison, the $F_{\text {ICF }}$ predictions from Ref. [12] are shown by open circles. While several changes were made to the determination of coincidence efficiencies and extraction of breakup probabilities, the total change in the breakup functions used as input for calculations of above-barrier $F_{\text {ICF }}$ was relatively modest, as already discussed. Therefore, the changes to PLATYPUS to model breakup of ${ }^{8} \mathrm{Be}$ through the $2^{+}$resonance are the major drivers towards the observed reduction of $F_{\text {ICF }}$ by a factor of 2-3 relative to Ref. [12].

Experimentally, complete fusion suppression has been deduced, independently of $\sigma_{\mathrm{ICF}}$, through comparison with reactions forming the same compound nucleus involving only well-bound nuclei $[2,6]$. Within the classical dynamical model followed in PLATYPUS, $F_{\text {ICF }}$ and complete fusion suppression are directly related, except some impact parameters outside the grazing trajectory that can only contribute to $\sigma_{\mathrm{ICF}}$ and not to $\sigma_{\mathrm{CF}}$. To demonstrate that such trajectories do not make a significant contribution to $\sigma_{\text {ICF }}$, we performed calculations with PLATYPUS switching off breakup. The resulting fusion cross section $\sigma_{\text {fus }}^{\text {no }} \mathrm{BU}$ is compared with $\sigma_{\mathrm{CF}}^{\text {with } \mathrm{BU}}$ obtained with PLATYPUS. The quantity $\left(1-\sigma_{\mathrm{CF}}^{\text {with } \mathrm{BU}} / \sigma_{\text {fus }}^{\text {no }} \mathrm{BU}\right)$, shown by purple triangles in Fig. 9, is very close to $F_{\text {ICF. This demonstrates }}$ that contributions to $\sigma_{\text {ICF }}$ from trajectories outside the grazing trajectory are small.

To understand the specific role of lifetime in $F_{\text {ICF }}$ predictions, the lifetime of the $2^{+}$state was changed to be a factor 


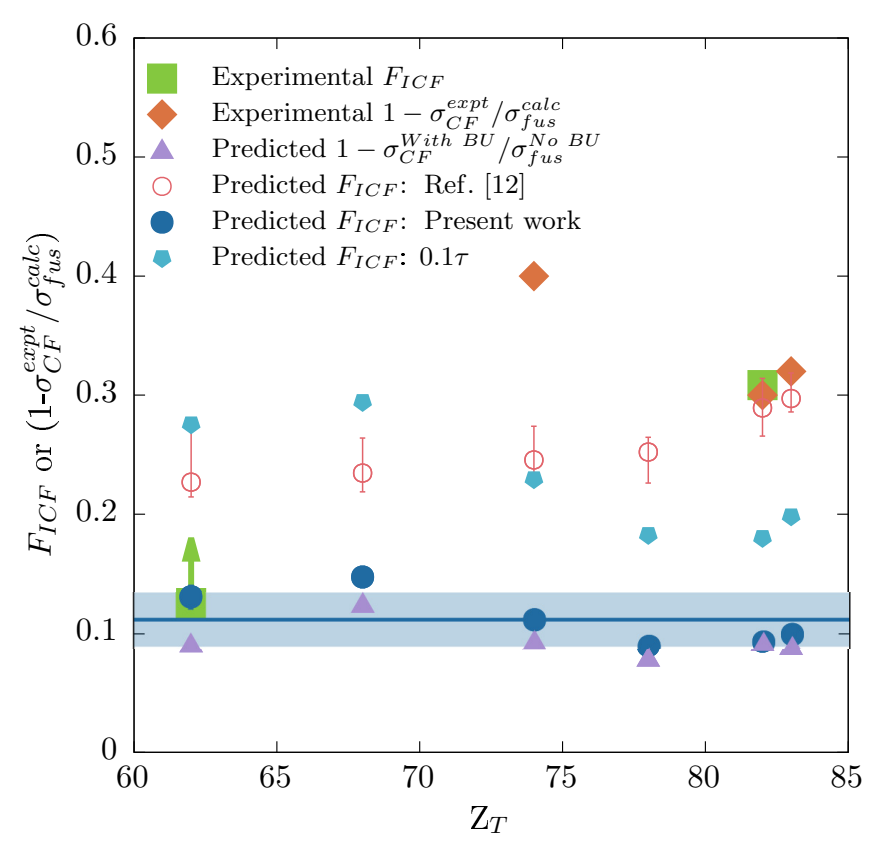

FIG. 9. Experimental values of $F_{\mathrm{ICF}}[4,7]$ (solid squares) and $1-\sigma_{\mathrm{CF}}^{\text {expt }} / \sigma_{\text {fus }}^{\text {calc }}[2,10]$ (solid diamonds), shown as a function of target $Z$. Predictions of $F_{\mathrm{ICF}}$ (solid circles) and complete fusion suppression (solid triangles) using the new breakup functions and the modified version of platypus. Error bars (determined from the uncertainty in the least-squares fit) are smaller than the points. The $F_{\text {ICF }}$ and complete fusion suppression predictions show no clear trend with $Z$. The $F_{\text {ICF }}$ prediction has a mean value of $0.11 \pm 0.02$, shown as the solid line, and the shaded bar indicates $\pm 1 \sigma .1-\sigma_{\mathrm{CF}}^{\text {with } \mathrm{BU}} / \sigma_{\text {fus }}^{\text {no } \mathrm{BU}}$ has a mean value of $0.09 \pm 0.02 . F_{\text {ICF }}$ predictions made using the lifetime of the $2^{+}$state ten times smaller than expected are shown with pentagons. $F_{\text {ICF }}$ predictions from Ref. [12] are shown with open circles.

of ten smaller. The results are shown by the blue pentagons in Fig. 9 and are typically a factor of two larger than previously (blue circles). This result makes the importance of explicit handling of lifetimes very clear. Indeed, the experimentally measured $\theta_{12}-\beta$ distributions compared to PLATYPUS simulations, shown in Fig. 5, already indicates that at below-barrier energies, the explicit inclusion of lifetimes change the breakup observables.

Experimental measurements of $F_{\text {ICF }}$ (which include any contributions from transfer) are shown in Fig. 9 as solid squares for ${ }^{9} \mathrm{Be}+{ }^{208} \mathrm{~Pb}$ [2] and ${ }^{144} \mathrm{Sm}$ [7]. For $F_{\text {ICF }}$ measurements to be made, both $\mathrm{CF}$ and ICF cross sections must be measured. However, because both $\mathrm{CF}$ and ICF cross sections are unavailable, fusion suppression factors $1-\sigma_{\mathrm{CF}}^{\text {expt }} / \sigma_{\text {fus }}^{\text {calc }}$ are shown for ${ }^{9} \mathrm{Be}+{ }^{209} \mathrm{Bi}$ [4] and ${ }^{186} \mathrm{~W}$ [10] as diamonds in Fig. 9. As both $F_{\mathrm{ICF}}$ and the fusion suppression factor are available for ${ }^{9} \mathrm{Be}+{ }^{208} \mathrm{~Pb}$ [2], both are shown, demonstrating the agreement between both quantities in this system. The measured $F_{\mathrm{ICF}}$ and fusion suppressions for ${ }^{9} \mathrm{Be}+{ }^{209} \mathrm{Bi}$ and ${ }^{208} \mathrm{~Pb}$ are a factor of three times larger than the predicted contribution from neutron-transfer-triggered breakup, and the experimental fusion suppression determined for ${ }^{9} \mathrm{Be}+{ }^{186} \mathrm{~W}$ is a factor of four times larger. The $F_{\mathrm{ICF}}$ determined for ${ }^{9} \mathrm{Be}+{ }^{144} \mathrm{Sm}$ is consistent with the prediction. However, the measured ICF cross section in this experiment represents a lower limit because cross sections for ${ }^{146} \mathrm{Gd}$ and ${ }^{148} \mathrm{Gd}$ were not included [7]. Further, as indicated in Fig. 9, even with lifetimes that are a factor of ten smaller than those estimated from the width of the $2^{+}$resonance in ${ }^{8} \mathrm{Be}$, the predicted $F_{\text {ICF }}$ resulting from breakup cannot be reconciled with the experimentally measured suppression of complete fusion for ${ }^{9} \mathrm{Be}+{ }^{186} \mathrm{~W}$, ${ }^{208} \mathrm{~Pb}$ and ${ }^{209} \mathrm{Bi}$.

\section{CONCLUSIONS}

Explicit inclusion of excitation energies and lifetimes of unbound resonances are crucial to model breakup. In the absence of a quantum mechanical model of transfer-triggered breakup, they have been included by modifying the classical dynamical code PLATYPUS. The new calculations show improved agreement with the measured energy and angular correlations of the breakup fragments. These correlations show sensitivity even to the subzeptosecond lifetimes of the $2^{+}$ state of ${ }^{8} \mathrm{Be}$ formed following $n$ transfer from ${ }^{9} \mathrm{Be}$. Above the barrier, the inclusion of these lifetimes significantly reduces predicted above-barrier suppression of complete fusion. This occurs because a larger fraction of nuclei remain intact until reaching the barrier. As a result, predicted complete fusion cross sections are not suppressed to the extent expected from earlier calculations that do not explicitly include lifetimes. This result is expected to apply to weakly bound nuclei in general.

To make quantitative predictions of complete fusion suppression at above-barrier energies, breakup probabilities extracted from the experiments were used as input to the modified version of PLATYPUS that explicitly includes lifetime effects. This results in incomplete fusion to total fusion fractions $F_{\text {ICF }}$ of $\sim 11 \%$ at above-barrier energies. The related complete fusion suppression of $\sim 9 \%$ is much less than the experimentally measured $F_{\text {ICF }}$ and complete fusion suppressions of $30 \%-40 \%[2,4,10]$.

Three key conclusions are drawn from these results:

(1) As the calculated $F_{\text {ICF }}$ is much less than measured, the cross sections that are attributed experimentally to ICF may include a significant contribution from transfer directly producing the same heavy nucleus. This needs to be investigated in more detail.

(2) If $\sigma_{\text {ICF }}$ contains contributions from both ICF and transfer, defining an empirical complete fusion suppression $F_{\text {ICF }}$ in terms of $\sigma_{\text {ICF }}$ is problematic.

(3) The observed reduction of complete fusion at abovebarrier energies has been measured independently of $\sigma_{\text {ICF }}$ in several reactions through direct comparison with reactions of well-bound nuclei $[2,6]$. Because breakup cannot explain this, other processes must contribute. Experimental values of $F_{\mathrm{ICF}}$ and $1-\sigma_{\mathrm{CF}}^{\text {expt }} / \sigma_{\text {fus }}^{\text {calc }}$ have been found to be similar [2]; thus, it is reasonable to suspect that the two quantities are linked. Therefore, if transfer is shown to make a large contribution to products previously attributed to ICF, then a mechanism by which transfer may suppress complete fusion needs to be considered. In a classical picture, if transfer removes energy from the relative motion, it will reduce fusion. 
However, in a coupled-channels approach, it is not clear whether above-barrier fusion can be suppressed by transfer. These questions require further investigation.

\section{ACKNOWLEDGMENTS}

This work was supported by ARC Grants No. FL110100098, No. DP130101569, and No. DP140101337. Support for ANU Heavy Ion Accelerator Facility accelerator operations through the NCRIS program is acknowledged. Helpful discussions with A. Diaz-Torres and R. Rafiei are gratefully acknowledged. We thank R. du Rietz and M. Evers for their assistance in running the original experiment.

\section{APPENDIX A: REMOVAL OF CROSS-TALK EVENTS}

In the previous work, spurious coincident events resulting from charge sharing across adjacent pixels resulting from cross talk or particles crossing the interstrip partition were removed by rejecting any event in adjacent pixels. With greater experience in analysis of such data, it was realized that these events can be rejected by their unphysical relative energy $\left(E_{\mathrm{rel}}\right)$ with respect to their opening angle $\left(\theta_{12}\right)$. In this analysis, spurious events were removed by applying cuts in the $E_{\mathrm{rel}}-\theta_{12}$ spectra. This alternate method for extracting breakup events resulted in an approximately four-times-larger yield of the ground-state ${ }^{8} \mathrm{Be}$ events because the vast majority of genuine ${ }^{8} \mathrm{Be}$ ground-state breakup events result in signals in adjacent pixels. These lost events would otherwise have had to be restored by a larger efficiency correction.

\section{APPENDIX B: NORMALIZATION}

The expected yield of Rutherford scattering $N_{\text {Ruth }}\left(\theta_{\text {Ruth }}\right)$ may be determined from the yield of Rutherford scattered particles $N_{\text {Ruth }}\left(\theta_{\text {norm }}\right)$ in the $\theta_{\text {norm }}=124^{\circ}$ to $127^{\circ}$ elastic normalization bin,

$$
N_{\text {Ruth }}\left(\theta_{\text {Ruth }}\right)=N_{\text {Ruth }}\left(\theta_{\text {norm }}\right) \frac{\left(\frac{d \sigma}{d \Omega}\right)\left(\theta_{\text {bin }}\right)}{\left(\frac{d \sigma}{d \Omega}\right)\left(\theta_{\text {norm }}\right)}\left(\frac{d \Omega_{\text {bin }}}{d \Omega_{\text {norm }}}\right),
$$

where $\frac{d \sigma}{d \Omega}\left(\theta_{x}\right)$ and $d \Omega\left(\theta_{x}\right)$ are the differential cross sections and solid angles, respectively. Because the efficiency-corrected breakup yield corresponds to the number of coincidence breakup events over all azimuthal angles, the calculated Rutherford yield must be for this same angular range. In Ref. [12] the Rutherford yield was calculated within the coverage of BALiN. This leads to a downwards correction in the present study by a factor of $\sim 0.75$, equal to the fractional coverage of the BALiN array in azimuthal angle.

Because $\theta_{\text {norm }}$ is at a relatively backwards angle, the elastic yield is purely Rutherford only for deep sub-barrier measurements. Where measurements were made near to the barrier, the expected $N_{\text {Ruth }}\left(\theta_{\text {norm }}\right)$ was calculated from the elastic yield, $N_{\text {elas }}\left(\theta_{\text {norm }}\right)$, by taking the ratio of the elastic and Rutherford cross sections determined from optical model fits of existing elastic scattering data [30-33], such that

$$
N_{\text {Ruth }}\left(\theta_{\text {norm }}\right)=N_{\text {elas }}\left(\theta_{\text {norm }}\right)\left(\frac{\frac{d \sigma_{\text {elas }}}{d \Omega}}{\frac{d \sigma_{\text {Ruth }}}{d \Omega}}\right)\left(\theta_{\text {norm }}\right) .
$$

The correction was largest for ${ }^{9} \mathrm{Be}+{ }^{208} \mathrm{~Pb}$ and ${ }^{209} \mathrm{Bi}$ at $E_{\text {beam }}=37 \mathrm{MeV}$, where $\frac{d \sigma_{\text {elas }} / d \Omega}{d \sigma_{\text {Ruth }} / d \Omega}\left(\theta_{\text {norm }}\right)=0.89$. The solid angle coverage of the normalization bin $d \Omega_{\text {norm }}$ can be determined from the solid angle coverage of BALiN by comparing the yields in normalization bin and in each $\theta$ bin of BALiN at a beam energy $E_{\text {cal }}$, where the elastic yields do not significantly deviate from Rutherford scattering for all angles. In that case, we can write

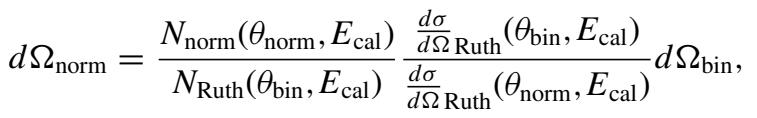

where $d \Omega_{\text {bin }}$ is the solid angle coverage for each $\theta$ bin in BALiN.

\section{APPENDIX C: EFFICIENCY DETERMINATION}

Using the notion of $\theta_{8} \mathrm{Be}$, the geometric coincidence efficiency was given by the ratio of simulated breakup events that would have landed in BALiN at each $\theta_{8}$ Be and $\theta_{12}$ (taking into account the azimuthal coverage of BALiN) to the simulated events distributed over all azimuthal angles. The simulated events were subject to the same detector conditions as the experimental data. As an example, the geometric coincidence efficiency matrix determined for ${ }^{8} \mathrm{Be}+{ }^{208} \mathrm{~Pb}$ at $34.0 \mathrm{MeV}$ is shown in Fig. 10(c). This was determined from the ratio of the number of events within the acceptance of BALiN [Fig. 10(b)] to the total number of events [Fig. 10(a)] in each $\left(\theta_{{ }^{8} \mathrm{Be}}, \theta_{12}\right)$ bin. The experimentally determined $\left(\theta_{8} \mathrm{Be}, \theta_{12}\right)$ distribution for the same system is shown in Fig. 10(d). The geometric coincidence efficiency shows two triangular regions of high detector efficiency: at small $\theta_{12} \sim 10^{\circ}$ with $\theta^{8} \mathrm{Be} / 135^{\circ}$ corresponding to the center of the BALiN array and at $\theta_{12} \sim 80^{\circ}$ at backward $\theta_{8 \mathrm{Be}} \sim 180^{\circ}$. The former is due to events with sufficiently small opening angle so that both fragments land on the same DSSD, while the latter is due to events that strike two different DSSDs. For values of $\theta^{8} \mathrm{Be}$ where BALiN gives coverage, for some values of $\theta_{12}$ the efficiency is zero. A correction to account for this is made in the next stage in the determination of the efficiency.

To simulate the distribution of fragments, shown Fig. 10(a), needed for this second part of the efficiency correction, PLATYPUS calculations using the modifications discussed in Sec. IV were performed. Simulations of near-target (high $\left.E_{\text {rel }}\right)$ breakup events were performed using the excitation energy and excitation energy dependent mean life for ${ }^{8} \mathrm{Be}$ $2^{+}$as discussed in Sec. IV. $Q$-value distributions were taken from the experimental results and the energy of the ${ }^{8} \mathrm{Be}$ pseudoprojectile $\left(E_{\mathrm{P}}^{\prime}\right)$ calculated by matching the distance of closest approach to that attained by the ${ }^{9} \mathrm{Be}$ beam with energy $E_{\mathrm{P}}$ because PLATYPUS does not simulate transfer. In analogy to the optimum $Q$ value of Ref. [34], this matching energy is given by

$$
E_{\mathrm{P}}^{\prime}=E_{\mathrm{P}} \frac{m_{\mathrm{T}}}{m_{\mathrm{T}}^{\prime}}\left(\frac{Z_{\mathrm{P}} Z_{\mathrm{T}}}{Z_{\mathrm{P}}^{\prime} Z_{\mathrm{T}}^{\prime}}\right),
$$

where $Z_{\mathrm{T}}, m_{\mathrm{T}}, Z_{\mathrm{P}}, m_{\mathrm{P}}$ and $Z_{\mathrm{T}}^{\prime}, m_{\mathrm{T}}^{\prime}, Z_{\mathrm{P}}^{\prime}, m_{\mathrm{P}}^{\prime}$ is the charge and mass of the target and projectile before and after transfer, 

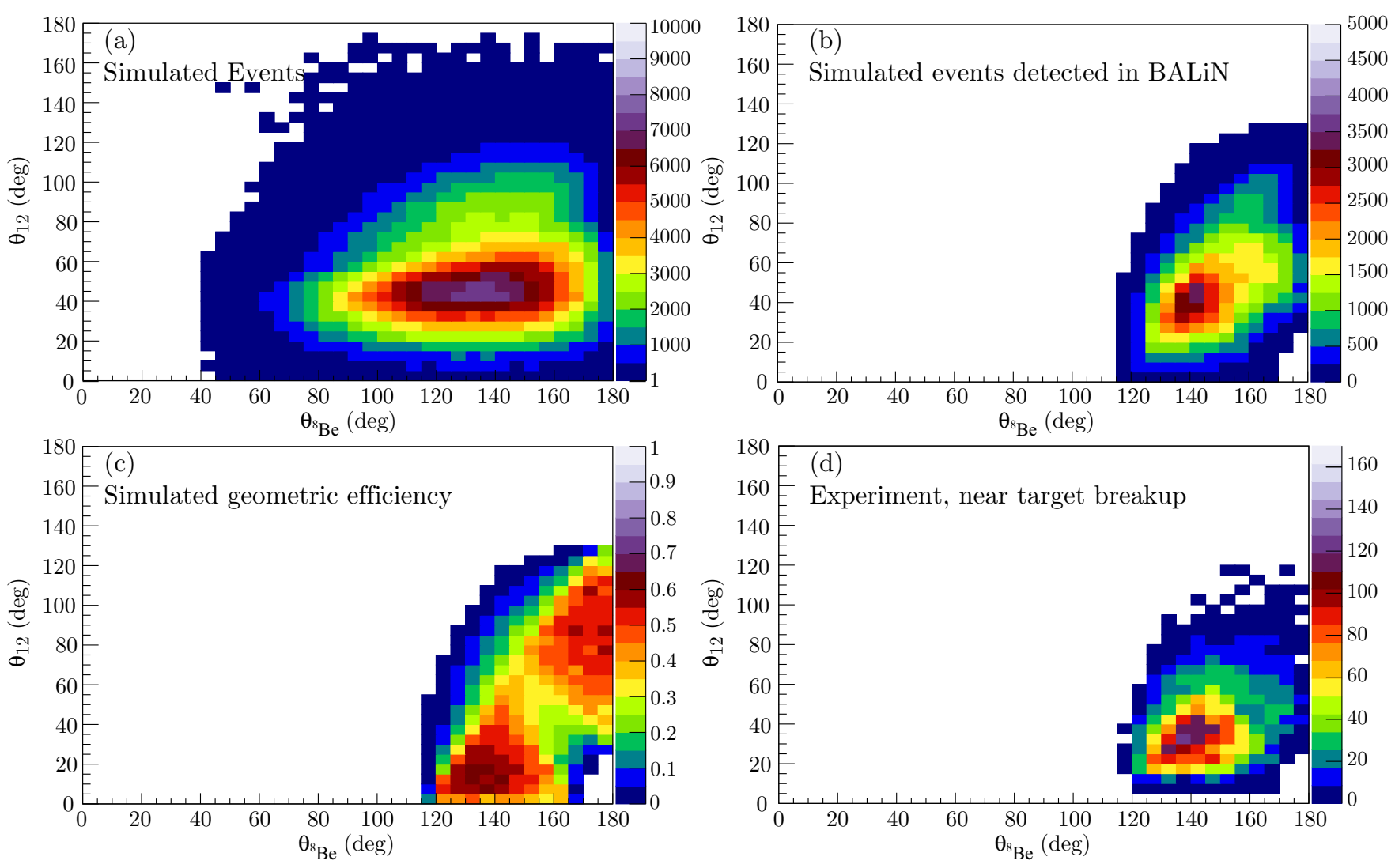

FIG. 10. Simulated and experimental near-target $\theta_{8_{\mathrm{Be}}}-\theta_{12}$ distributions for ${ }^{8} \mathrm{Be}_{2^{+}}+{ }^{208} \mathrm{~Pb} \rightarrow \alpha+\alpha+{ }^{207} \mathrm{~Pb}$ at $E_{\text {beam }}=34 \mathrm{MeV}$. (a) Total simulated distribution. (b) The same events filtered by the acceptance of the BALiN array. (c) The associated geometric coincidence efficiency of the BALiN array determined from the simulated events filtered by the acceptance of BALiN divided by the total simulated events in each $\left(\theta_{8_{\mathrm{Be}}}, \theta_{12}\right)$ bin. (d) Experimental $\theta_{8_{\mathrm{Be}}}-\theta_{12}$ distribution for near-target breakup events [region (ii) of Fig. 1] showing the good correspondence between the filtered simulated data and the experiment

respectively. In this case, where only neutron transfer is occurring, the matching energy is very close to the experimental beam energy. In cases such as the breakup of ${ }^{7} \mathrm{Li}$, where proton transfer dominates, this factor becomes more important. The projectile-target and fragment-target potentials are Woods-Saxon parametrizations of São Paulo potentials [29] from Ref. [12]. This is used for all PLATYPUS simulations in this work. According to these simulations, events that have $\theta^{8} \mathrm{Be}$ where BALiN gives coverage but have $\theta_{12}$ where the efficiency is zero accounted for $\sim 7 \%$ of all events simulated within the $\theta_{8} \mathrm{Be}$ acceptance of the array. As such, this second step in efficiency correction represents a small (though $\theta_{8}{ }_{\mathrm{Be}}$-dependant) model-dependent addition to a model-independent efficiency correction.

\section{APPENDIX D: COMPARISON OF EFFICIENCIES IN REF. [12] AND THE PRESENT WORK}

The coincidence efficiencies determined in this work differ from those found in Ref. [12]: there, prompt efficiencies were given for two $15^{\circ}$ bins in $\theta^{8} \mathrm{Be}, \epsilon_{121^{\circ}-136^{\circ}}=0.25$ and $\epsilon_{136^{\circ}-151^{\circ}}=0.42$, and the efficiencies were found to be nearly independent of $E_{\text {lab. }}$. Here the efficiencies are calculated in $5^{\circ}$ bins in $\left(\theta_{12}, \theta^{8} \mathrm{Be}\right)$ and so are much more fine grained. However, when averaged over the same range of $\theta_{8} \mathrm{Be}$, the efficiencies in this work are, on average, $\epsilon_{121^{\circ}-136^{\circ}}=0.17$ and $\epsilon_{136^{\circ}-151^{\circ}}=0.40$. As the efficiency corrections in this work take into account $\theta_{12}$, the distribution of which changes with $E_{\text {lab }}$ and $Z$, these averaged efficiencies are not independent of $E_{\text {lab }}$ or $Z$.

These differences between Ref. [12] and the present work can be accounted by three factors. (i) In the previous analysis, efficiencies were calculated as a function only of $\theta_{8} \mathrm{Be}$. As seen in Fig. 10(c), for events with a $\left(\theta_{12}, \theta_{{ }_{8} \mathrm{Be}}\right)$ distribution as shown in Fig. 10(a), the efficiency varies strongly as a function of $\theta_{12}$ for fixed $\theta_{8} \mathrm{Be}$. Thus, efficiency correction only as a function of $\theta_{8} \mathrm{Be}$ results in an average overcorrection in the number of breakup pairs for each $\theta^{8} \mathrm{Be}$ by a factor of $\sim 1.1$ for the systems studied in this work (depending on $\theta_{8} \mathrm{Be}$, target mass, and beam energy), compared to the new two-dimensional efficiency correction performed here. (ii) The efficiencies further change as the early version of PLATYPUS used in Ref. [12] did not have a fully isotropic distribution of initial fragment directions: There was an overabundance of events with similar scattering angles, $\theta_{1} \sim \theta_{2}$, leading to an artificially high efficiency. PLATYPUS was corrected in late 2010 [35]. (iii) The modifications of PLATYPUS performed for this work resulted in a different angular distribution of fragments and so changed the model-dependent stage of the efficiency corrections. 


\section{APPENDIX E: LOCAL BREAKUP PROBABILITIES}

Because interacting nuclei spend more time near the distance of closest approach, then casting the breakup probability as

$$
\frac{d P}{d r} \propto e^{\mu r}
$$

in a dynamical model is inappropriate. To illustrate this, consider a classical Coulomb trajectory, where

$$
\frac{d t}{d r}=\frac{r}{v \sqrt{\left[r-a_{0}(1+\epsilon)\right]\left[r-a_{0}(1-\epsilon)\right]}},
$$

and $a_{0}=Z_{p} Z_{t} e^{2} / \mu v^{2}, \epsilon=\sqrt{1+(L / \eta)^{2}}$, and the Sommerfeld parameter $\eta=Z_{p} Z_{t} e^{2} / v$, where $\mu$ is the reduced mass and $v$ the incident velocity. Then

$$
\frac{d P}{d t}=\frac{d P}{d r} \frac{d r}{d t} \propto e^{-\mu r} \frac{v \sqrt{\left[r-a_{0}(1+\epsilon)\right]\left[r-a_{0}(1-\epsilon)\right]}}{r} .
$$

For a trajectory corresponding to scattering at $180^{\circ}, \epsilon=1$, and the distance of closest approach, $R_{0}=2 a_{0}$, this results in $d P\left(R_{\min }\right) / d t=0$ at the distance of closest approach, which does not seem reasonable.
[1] M. Dasgupta, D. J. Hinde, R. D. Butt, R. M. Anjos, A. C. Berriman, N. Carlin, P. R. S. Gomes, C. R. Morton, J. O. Newton, A. Szanto de Toledo, and K. Hagino, Phys. Rev. Lett. 82, 1395 (1999).

[2] M. Dasgupta, P. R. S. Gomes, D. J. Hinde, S. B. Moraes, R. M. Anjos, A. C. Berriman, R. D. Butt, N. Carlin, J. Lubian, C. R. Morton, J. O. Newton, and A. Szanto de Toledo, Phys. Rev. C 70, 024606 (2004).

[3] C. Signorini, T. Glodariu, Z. H. Liu, M. Mazzocco, M. Ruan, and F. Soramel, Prog. Theor. Phys. Suppl. 154, 272 (2004).

[4] M. Dasgupta, D. J. Hinde, S. L. Sheehy, and B. Bouriquet, Phys. Rev. C 81, 024608 (2010).

[5] M. Dasgupta, D. J. Hinde, K. Hagino, S. B. Moraes, P. R. S. Gomes, R. M. Anjos, R. D. Butt, A. C. Berriman, N. Carlin, C. R. Morton, J. O. Newton, and A. Szanto de Toledo, Phys. Rev. C 66, 041602(R) (2002).

[6] P. K. Rath, S. Santra, N. L. Singh, R. Tripathi, V. V. Parkar, B. K. Nayak, K. Mahata, R. Palit, S. Kumar, S. Mukherjee, S. Appannababu, and R. K. Choudhury, Phys. Rev. C 79, 051601 (2009).

[7] P. R. S. Gomes, I. Padron, E. Crema, O. A. Capurro, J. O. Fernández Niello, A. Arazi, G. V. Martí, J. Lubian, M. Trotta, A. J. Pacheco, J. E. Testoni, M. D. Rodríguez, M. E. Ortega, L. C. Chamon, R. M. Anjos, R. Veiga, M. Dasgupta, D. J. Hinde, and K. Hagino, Phys. Rev. C 73, 064606 (2006).

[8] C. S. Palshetkar, S. Santra, A. Chatterjee, K. Ramachandran, S. Thakur, S. K. Pandit, K. Mahata, A. Shrivastava, V. V. Parkar, and V. Nanal, Phys. Rev. C 82, 044608 (2010).

[9] V. V. Parkar, R. Palit, S. K. Sharma, B. S. Naidu, S. Santra, P. K. Joshi, P. K. Rath, K. Mahata, K. Ramachandran, T. Trivedi, and A. Raghav, Phys. Rev. C 82, 054601 (2010).

[10] Y. D. Fang, P. R. S. Gomes, J. Lubian, X. H. Zhou, Y. H. Zhang, J. L. Han, M. L. Liu, Y. Zheng, S. Guo, J. G. Wang, Y. H. Qiang, Z. G. Wang, X. G. Wu, C. Y. He, Y. Zheng, C. B. Li, S. P. Hu, and S. H. Yao, Phys. Rev. C 87, 024604 (2013).

[11] D. J. Hinde, M. Dasgupta, B. R. Fulton, C. R. Morton, R. J. Wooliscroft, A. C. Berriman, and K. Hagino, Phys. Rev. Lett. 89, 272701 (2002).

[12] R. Rafiei, R. du Rietz, D. H. Luong, D. J. Hinde, M. Dasgupta, M. Evers, and A. Diaz-Torres, Phys. Rev. C 81, 024601 (2010).

[13] D. H. Luong, M. Dasgupta, D. J. Hinde, R. Du Rietz, R. Rafiei, C. J. Lin, M. Evers, and A. Diaz-Torres, Phys. Lett. B 695, 105 (2011).
[14] D. R. Tilley, J. H. Kelley, J. L. Godwin, D. J. Millener, J. E. Purcell, C. G. Sheu, and H. R. Weller, Nucl. Phys. A 745, 155 (2004).

[15] A. Diaz-Torres, D. J. Hinde, J. A. Tostevin, M. Dasgupta, and L. R. Gasques, Phys. Rev. Lett. 98, 152701 (2007).

[16] A. Diaz-Torres, Comput. Phys. Commun. 182, 1100 (2011).

[17] E. C. Simpson, K. J. Cook, D. H. Luong, S. Kalkal, I. P. Carter, M. Dasgupta, D. J. Hinde, and E. Williams, Phys. Rev. C 93, 024605 (2016).

[18] F. C. Barker and P. B. Treacy, Nucl. Phys. 38, 33 (1962).

[19] F. C. Barker, Aust. J. Phys. 41, 743 (1988).

[20] D. H. Luong, M. Dasgupta, D. J. Hinde, R. du Rietz, R. Rafiei, C. J. Lin, M. Evers, and A. Diaz-Torres, Phys. Rev. C 88, 034609 (2013)

[21] A. B. McIntosh, S. Hudan, C. J. Metelko, R. T. de Souza, R. J. Charity, L. G. Sobotka, W. G. Lynch, and M. B. Tsang, Phys. Rev. Lett. 99, 132701 (2007).

[22] S. Kalkal, E. C. Simpson, D. H. Luong, K. J. Cook, M. Dasgupta, D. J. Hinde, I. P. Carter, D. Y. Jeung, G. Mohanto, C. S. Palshetkar, E. Prasad, D. C. Rafferty, C. Simenel, K. Vo-Phuoc, E. Williams, L. R. Gasques, P. R. S. Gomes, and R. Linares, Phys. Rev. C 93, 044605 (2016).

[23] J. E. Mason, S. B. Gazes, R. B. Roberts, and S. G. Teichmann, Phys. Rev. C 45, 2870 (1992).

[24] L. Corradi, S. J. Skorka, U. Lenz, K. E. G. Löbner, P. R. Pascholati, U. Quade, K. Rudolph, W. Schomburg, M. Steinmayer, H. G. Theis, G. Montagnoli, D. R. Napoli, A. M. Stefanini, A. Tivelli, S. Beghini, F. Scarlassara, C. Signorini, and F. Soramel, Z. Phys. A 334, 55 (1990).

[25] J. Knoll and R. Schaeffer, Phys. Rep. 31, 159 (1977).

[26] E. Vigezzi and A. Winther, Ann. Phys. 192, 432 (1989).

[27] P. R. S. Gomes, R. Linares, J. Lubian, C. C. Lopes, E. N. Cardozo, B. H. F. Pereira, and I. Padron, Phys. Rev. C 84, 014615 (2011).

[28] V. Tripathi, A. Navin, V. Nanal, R. G. Pillay, K. Mahata, K. Ramachandran, A. Shrivastava, A. Chatterjee, and S. Kailas, Phys. Rev. C 72, 017601 (2005).

[29] L. C. Chamon, B. V. Carlson, L. R. Gasques, D. Pereira, C. De Conti, M. A. G. Alvarez, M. S. Hussein, M. A. Cândido Ribeiro, E. S. Rossi, and C. P. Silva, Phys. Rev. C 66, 014610 (2002).

[30] C. Signorini, A. Andrighetto, J. Y. Guo, M. Ruan, L. Stroe, F. Soramel, K. E. G. Löbner, L. Müller, D. Pierroutsakou, 
M. Romoli, K. Rudolph, I. Thompson, M. Trotta, and A. Vitturi, Nucl. Phys. A 701, 23 (2002).

[31] V. V. Parkar, V. Jha, S. K. Pandit, S. Santra, and S. Kailas, Phys. Rev. C 87, 034602 (2013).

[32] N. Yu, H. Q. Zhang, H. M. Jia, S. T. Zhang, M. Ruan, F. Yang, Z. D. Wu, X. X. Xu, and C. L. Bai, J. Phys. G: Nucl. Part. Phys. 37, 075108 (2010).
[33] V. I. Zagrebaev, A. S. Denikin, A. P. Alekseev, A. V. Karpov, V. V. Samarin, M. A. Naumenko, and A. Y. Kozhin, OM Code, Nuclear Reactions Video Project, http://nrv.jinr.ru/nrv/.

[34] J. P. Schiffer, H. J. Kijrner, R. H. Siemssen, K. W. Jones, and A. Schwarzschild, Phys. Lett. B. 44, 47 (1973)

[35] A. Diaz-Torres (private communication). 